\title{
Uniqueness and Optimality of Dynamical Extensions of Divergences
}

\author{
Gilad Gour* \\ Department of Mathematics and Statistics, Institute for Quantum Science and Technology, University of Calgary, \\ Alberta T2N 1N4, Canada
}

(Received 24 July 2020; revised 11 December 2020; accepted 22 December 2020; published 22 January 2021)

\begin{abstract}
We introduce an axiomatic approach for channel divergences and channel relative entropies that is based on three information-theoretic axioms of monotonicity under superchannels, i.e., generalized data processing inequality, additivity under tensor products, and normalization, similar to the approach given for the state domain in Gour and Tomamichel [arXiv:2006.11164v1 (2020), arXiv:2006.12408v2 (2020)]. We show that these axioms are sufficient to give enough structure in the channel domain as well, leading to numerous properties that are applicable to all channel divergences. These include faithfulness, continuity, a type of triangle inequality, and boundedness between the min and max channel relative entropies. In addition, we prove a uniqueness theorem showing that the Kullback-Leibler divergence has only one extension to classical channels. For quantum channels, with the exception of the max relative entropy, this uniqueness does not hold. Instead, we prove the optimality of the amortized channel extension of the Umegaki relative entropy, by showing that it provides a lower bound on all channel relative entropies that reduce to the Kullback-Leibler divergence on classical states. We also introduce the maximal channel extension of a given classical state divergence and study its properties.
\end{abstract}

DOI: 10.1103/PRXQuantum.2.010313

\section{INTRODUCTION}

Distinguishability between probability distributions, quantum states, and quantum channels lies at the heart of several theories in science, including information theory, quantum computing, and statistics. Unlike arbitrary vectors, objects such as probability vectors, quantum states, and quantum channels contain information about physical systems and therefore their distinguishability is typically quantified with functions that are sensitive to this information. For example, consider an information source (see, e.g., Ref. [1]) such as a dice, that "emits" alphabets in $\mathcal{X}$ according to either probability distribution $\mathbf{p}:=\left\{p_{x}\right\}_{x \in \mathcal{X}}$ or probability distribution $\mathbf{q}:=\left\{q_{x}\right\}_{x \in \mathcal{X}}$. An agent can estimate which of the two distributions corresponds to the source by using the source many times (in the example of a dice, this amounts to rolling it many times). The intuition is that if $\mathbf{p}$ and $\mathbf{q}$ are very distinguishable, it will be easier to determine which one of them corresponds to the information source.

One of the key observations in any distinguishability task as above is that by sending the information

\footnotetext{
*gour@ucalgary.ca
}

Published by the American Physical Society under the terms of the Creative Commons Attribution 4.0 International license. Further distribution of this work must maintain attribution to the author(s) and the published article's title, journal citation, and DOI. source through a communication channel, the agent cannot increase his or her ability to distinguish between the two distributions $\mathbf{p}$ and $\mathbf{q}$. This means that if $E$ is a column stochastic matrix that corresponds to the communication channel, then the resulting distributions $E \mathbf{p}$ and $E \mathbf{q}$ are less distinguishable than $\mathbf{p}$ and $\mathbf{q}$. In other words, any measure that quantifies the distinguishability between two probability vectors $\mathbf{p}$ and $\mathbf{q}$ must decrease under any stochastic evolution process that takes the pair $(\mathbf{p}, \mathbf{q})$ to $(E \mathbf{p}, E \mathbf{q})$. Hence, many metrics in $\mathbb{R}^{n}$, such as the Euclidean distance, cannot be used to quantify this notion of distinguishability between two probability vectors (see, e.g., Ref. [2]).

Functions defined on pairs of probabilities that behave monotonically under mapping of the form $(\mathbf{p}, \mathbf{q}) \rightarrow$ $(E \mathbf{p}, E \mathbf{q})$ are called divergences and their monotonicity property is often referred to as the data processing inequality (DPI). Unlike the trace distance [3], some divergences are additive under tensor products. In Refs. [4,5], relative entropies have been defined axiomatically as divergences that satisfy this additivity property. It has been shown that the combination of DPI with additivity provides enough structure to deduce a variety of key properties of relative entropies.

Many specific divergences and relative entropies have been extended to the quantum domain, including the trace distance, the Kullback-Leibler (KL) divergence, the Reńyi divergences, $f$ divergences, and the min and max relative entropies [6-13]. More recently, several extensions of quantum divergences to channel divergences have 
also been studied [14-19], with a variety of applications in resource theories of quantum channels and beyond [18-29]. In Ref. [5], an axiomatic approach to quantum divergences has been proposed. This approach is based on three axioms of DPI, additivity, and normalization that have first been put forth in Ref. [4] for classical divergences.

In this paper, we extend further this axiomatic approach to the channel domain. We define dynamical (i.e., channel) divergences as functions that satisfy the DPI with a superchannel and channel relative entropies as channel divergences that, in addition, satisfy additivity and appropriate normalization. We show that these axioms provide enough structure that give rises to a variety of properties that hold for all dynamical divergences and dynamical relative entropies. These include properties that carry over from the state domain, such as faithfulness, continuity, optimality, and the triangle inequality that holds for all channel relative entropies. We also show that all channel relative entropies must be no greater than the max channel relative entropy and no smaller than the min channel relative entropy. We then discuss a variety of extensions of classical (state) divergences to quantum-channel divergences. Remarkably, for classical channels, we find that

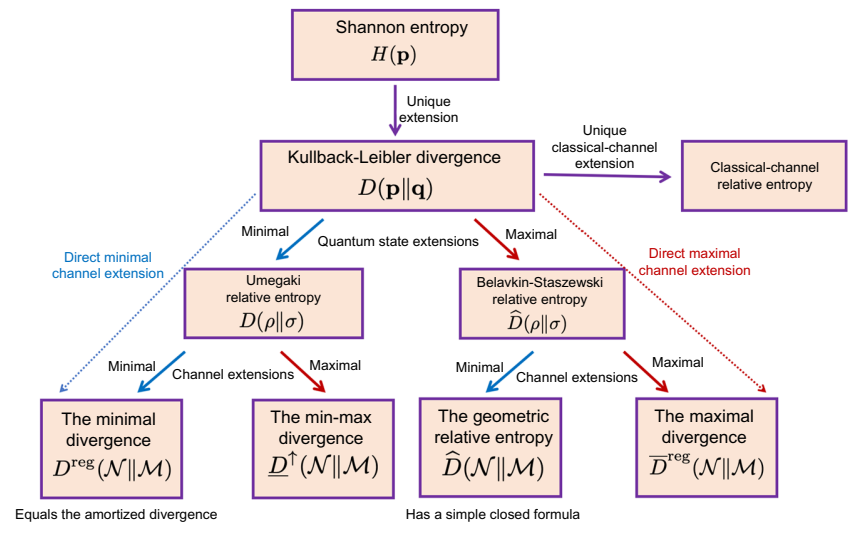

FIG. 1. A summary of all optimal extensions of the Shannon entropy. The red arrows represent maximal extensions and the blue arrows represent minimal extensions. The Kullback-Leibler divergence is the only classical relative entropy that reduces to the $\log$ of the dimension minus the Shannon entropy when the second argument is a uniform distribution (in Ref. [4], a oneto-one correspondence between classical entropies and classical relative entropies has been proven). Regularization is assumed in all extensions. The channel relative entropy $D^{\text {reg }}(\mathcal{N} \| \mathcal{M})$ equals both the amortized divergence and the regularized minimal channel extension $\underline{D}^{\text {reg }}(\mathcal{N} \| \mathcal{M})$. It is the smallest of all the channel relative entropies that reduce to the Kullback-Leibler divergence on classical states, while the maximal divergence $\bar{D}^{\text {reg }}(\mathcal{N} \| \mathcal{M})$ is the largest one. The blue and red dashed arrows indicate that the minimal and maximal channel extensions can be obtained directly from the Kullback-Leibler divergence using the extension techniques introduced in this paper. there exists only one classical-channel relative entropy that reduces to the KL divergence. In other words, the extension of the KL relative entropy to classical channels is unique. In the quantum domain, we show that the regularized channel (Umegaki) relative entropy is the smallest extension among all dynamical relative entropies that reduces to the KL divergence on classical states. In Fig. 1, we summarize all the optimal extensions of the Shannon entropy that we discuss in this paper. Similar figures can also be constructed for the Renyi entropies.

The paper is structured as follows. In Sec. II, we discuss the preliminaries material and the notation that is used throughout the paper. We then discuss the main results in Secs. III and IV and conclude with a summary and applications in Sec. V. The details of all proofs can be found in the Supplemental Material [30].

\section{PRELIMINARIES}

\section{A. Notation}

We denote both physical systems and their corresponding Hilbert spaces by $A, B, C$, etc. The letters $X, Y$, and $Z$ are reserved for classical systems. We only consider finitedimensional systems and we denote their dimensions by $|A|,|B|$, etc. The algebra of all $|A| \times|A|$ complex matrices is denoted by $\mathfrak{L}(A)$. Similarly, $\mathfrak{L}(X)$ denotes the algebra of all $|X| \times|X|$ diagonal matrices. The set of density matrices (i.e., quantum states) in $\mathfrak{L}(A)$ is denoted by $\mathfrak{D}(A)$. The elements of $\mathfrak{D}(A)$ are denoted by lowercase Greek letters such as $\rho, \sigma, \omega$, etc., whereas for a classical system $X$, the elements of the classical density matrices in $\mathfrak{D}(X)$ are denoted by $\mathbf{p}, \mathbf{q}, \mathbf{r}$, etc., where, depending on the context, $\mathbf{p}, \mathbf{q}$, and $\mathbf{r}$ are viewed as either diagonal density matrices in a fixed (classical) basis or probability vectors. Moreover, for classical systems, we sometimes use the notation $\mathfrak{D}(n) \equiv \mathfrak{D}(X)$ if $n=|X|$. The support of a density matrix $\rho$ is denoted by $\operatorname{supp}(\rho)$.

The set of all linear transformations from $\mathfrak{L}(A)$ to $\mathfrak{L}(B)$ is denoted by $\mathfrak{L}(A \rightarrow B)$. The elements of $\mathfrak{L}(A \rightarrow B)$ are denoted by calligraphic letters such as $\mathcal{N}, \mathcal{M}, \mathcal{E}, \mathcal{F}$, etc. The set of completely positive maps in $\mathfrak{L}(A \rightarrow B)$ is denoted by $\mathrm{CP}(A \rightarrow B)$ and the set of all completely positive trace-preserving maps (i.e., quantum channels) by $\operatorname{CPTP}(A \rightarrow B)$. We denote by 1 the trivial physical system and identify $\mathfrak{D}(A)=\operatorname{CPTP}(1 \rightarrow A)$. In particular, the set containing only the number 1 is identified with $\{1\}=$ $\mathfrak{D}(1)=$ CPTP $(1 \rightarrow 1)$. Note also that with these identifications, the trace is the only element of $\operatorname{CPTP}(A \rightarrow 1)=$ $\left\{\operatorname{Tr}_{A}\right\}$. The Choi matrix of a linear map $\mathcal{N} \in \mathfrak{L}(A \rightarrow B)$ is denoted by $J_{\mathcal{N}}^{A B}:=\sum_{j, k}|j\rangle\langle k| \otimes \mathcal{N}(|j\rangle\langle k|)$. For two linear maps $\mathcal{N}, \mathcal{M} \in \mathfrak{L}(A \rightarrow B)$, we write $\mathcal{N} \geq \mathcal{M}$ if $\mathcal{N}-$ $\mathcal{M} \in \mathrm{CP}(A \rightarrow B)$.

We also consider linear maps from $\mathfrak{L}(A \rightarrow B)$ to $\mathfrak{L}\left(A^{\prime} \rightarrow B^{\prime}\right)$. We call such linear maps supermaps and denote by $\mathbb{L}\left(A B \rightarrow A^{\prime} B^{\prime}\right)$ the set of all supermaps from 
$\mathfrak{L}(A \rightarrow B)$ to $\mathfrak{L}\left(A^{\prime} \rightarrow B^{\prime}\right)$. We also make the identification $\mathbb{L}(1 A \rightarrow 1 B)=\mathfrak{L}(A \rightarrow B)$, where, again, 1 denotes the trivial system. The elements of $\mathbb{L}\left(A B \rightarrow A^{\prime} B^{\prime}\right)$ are denoted by capital Greek letters such as $\Theta$ and $\Upsilon$. A supermap $\Theta \in$ $\mathbb{L}\left(A B \rightarrow A^{\prime} B^{\prime}\right)$ is called a superchannel if there exists a reference system $R$, a preprocessing map $\mathcal{E} \in \operatorname{CPTP}\left(A^{\prime} \rightarrow\right.$ $A R)$, and a postprocessing map $\mathcal{F} \in \operatorname{CPTP}\left(B R \rightarrow B^{\prime}\right)$ such that, for any $\mathcal{N} \in \mathfrak{L}(A \rightarrow B)$,

$$
\Theta\left[\mathcal{N}^{A \rightarrow B}\right]=\mathcal{F}^{B R \rightarrow B^{\prime}} \circ \mathcal{N}^{A \rightarrow B} \circ \mathcal{E}^{A^{\prime} \rightarrow A R} .
$$

In Ref. [31], it has been shown that a supermap is a superchannel if and only if it maps quantum channels to quantum channels in a complete sense (i.e., even when tensored with the identity supermap; for more details, see Refs. [17,27,32]). The set of all superchannels in $\mathbb{L}\left(A B \rightarrow A^{\prime} B^{\prime}\right)$ is denoted by $\mathfrak{S}\left(A B \rightarrow A^{\prime} B^{\prime}\right)$. We also use the notation

$$
\mathfrak{S}\left(A \rightarrow A^{\prime} B^{\prime}\right):=\mathfrak{S}\left(1 A \rightarrow A^{\prime} B^{\prime}\right)
$$

to denote superchannels that map quantum states in $\mathfrak{D}(A)=\operatorname{CPTP}(1 \rightarrow A)$ to quantum channels in CPTP $\left(A^{\prime} \rightarrow B^{\prime}\right)$. Similarly, $\mathfrak{S}\left(A B \rightarrow A^{\prime}\right)$ denotes the set of all superchannels that map channels in $\operatorname{CPTP}(A \rightarrow B)$ to states in $\mathfrak{D}\left(A^{\prime}\right)$.

\section{B. Divergences}

We follow the definition given in Refs. [4,5] of classical and quantum divergences and relative entropies.

Definition: [4,5] Let D : $\bigcup_{A}\{\mathfrak{D}(A) \times \mathfrak{D}(A)\} \rightarrow \mathbb{R} \cup$ $\{\infty\}$ be a function acting on pairs of quantum states in all finite dimensions.

1. The function $\mathbf{D}$ is called a divergence if it satisfies the DPI

$$
\mathbf{D}(\mathcal{E}(\rho) \| \mathcal{E}(\sigma)) \leq \mathbf{D}(\rho \| \sigma),
$$

for all $\mathcal{E} \in \operatorname{CPTP}(A \rightarrow B)$ and all $\rho, \sigma \in \mathfrak{D}(A)$.

2. A divergence $\mathbf{D}$ is called a relative entropy if, in addition, it satisfies:

(a) Normalization.

$$
\mathbf{D}\left(\left[\begin{array}{ll}
1 & 0 \\
0 & 0
\end{array}\right] \|\left[\begin{array}{cc}
1 / 2 & 0 \\
0 & 1 / 2
\end{array}\right]\right)=1 .
$$

(b) Additivity. For any $\rho_{1}, \rho_{2} \in \mathfrak{D}(A)$ and any $\sigma_{1}, \sigma_{2} \in$ $\mathfrak{D}(B)$

$$
\mathbf{D}\left(\rho_{1} \otimes \sigma_{1} \| \rho_{2} \otimes \sigma_{2}\right)=\mathbf{D}\left(\rho_{1} \| \sigma_{1}\right)+\mathbf{D}\left(\rho_{2} \| \sigma_{2}\right) .
$$

We use the bold D notation to denote both a general quantum divergence and a general quantum relative entropy, depending on the context. Similarly, we use the notation $\mathscr{D}$ to denote a general classical divergence or a general classical relative entropy. To denote specific divergences, we use the standard notation. For example, the classical Rényi divergence of order $\alpha \in[0, \infty]$ is denoted by

$$
D_{\alpha}(\mathbf{p} \| \mathbf{q}):=\frac{1}{\alpha-1} \log \sum_{x=1}^{|X|} p_{x}^{\alpha} q_{x}^{1-\alpha} \quad \forall \mathbf{p}, \mathbf{q} \in \mathfrak{D}(X) .
$$

The min and max quantum relative entropies [33] are denoted for any $\rho, \sigma \in \mathfrak{D}(A)$ by

$$
D_{\max }(\rho \| \sigma):= \begin{cases}\log \min \{t \geq 0: t \sigma \geq \rho\}, & \text { if } \rho \ll \sigma \\ \infty, & \text { otherwise }\end{cases}
$$

where $\rho \ll \sigma$ stands for $\operatorname{supp}(\rho) \subseteq \operatorname{supp}(\sigma)$, and

$$
D_{\min }(\rho \| \sigma):= \begin{cases}-\log \operatorname{Tr}\left[\sigma \Pi_{\rho}\right], & \text { if } \operatorname{Tr}[\rho \sigma] \neq 0 \\ \infty, & \text { otherwise }\end{cases}
$$

where $\Pi_{\rho}$ denotes the projection to the support of $\rho$ and $\rho \ll \sigma$ denotes $\operatorname{supp}(\rho) \subseteq \operatorname{supp}(\sigma)$. In Ref. [5] it has been shown that any quantum relative entropy $\mathbf{D}$ satisfies

$$
D_{\min }(\rho \| \sigma) \leq \mathbf{D}(\rho \| \sigma) \leq D_{\max }(\rho \| \sigma) \quad \forall \rho, \sigma \in \mathfrak{D}(A)
$$

The hypothesis-testing divergence can be viewed as the smoothed version of $D_{\min }$. It is given for all $\rho, \sigma \in \mathfrak{D}(A)$ by

$D_{\min }^{\epsilon}(\rho \| \sigma):=-\log \min$

$\left\{\operatorname{Tr}[\sigma E]: 0 \leq E \leq I^{A}, \quad \operatorname{Tr}[\rho E] \geq 1-\epsilon, \quad E \in \mathfrak{L}(A)\right\}$.

It can easily be verified that $D_{\min }^{\varepsilon=0}(\rho \| \sigma)=D_{\min }(\rho \| \sigma)$.

Another important example of a quantum relative entropy with several operational interpretations is the Umegaki relative entropy, given by

$$
D(\rho \| \sigma):=\operatorname{Tr}[\rho \log \rho]-\operatorname{Tr}[\rho \log \sigma] .
$$

This divergence plays a key role in quantum statistics [34], quantum Shannon theory [3], and quantum resource theories [35]. In Ref. [36] (see also Refs. [5,37]), it has been shown to be the only relative entropy that is asymptotically continuous. The Umegaki relative entropy is also known to be the regularized minimal quantum extension of the 
classical KL divergence, that is, for all $\rho, \sigma \in \mathfrak{D}(A)$

$$
D(\rho \| \sigma)=\underline{\mathrm{D}}^{\mathrm{reg}}(\rho \| \sigma):=\lim _{n \rightarrow \infty} \frac{1}{n} \underline{\mathrm{D}}\left(\rho^{\otimes n} \| \sigma^{\otimes n}\right),
$$

with

$$
\underline{\mathrm{D}}(\rho \| \sigma):=\sup _{\mathcal{E} \in \operatorname{CPTP}(A \rightarrow X)} D_{K L}[\mathcal{E}(\rho) \| \mathcal{E}(\sigma)],
$$

where $D_{K L}$ is the classical KL divergence and the supremum is over all classical systems $X$ and all positive operator-valued measures (POVMs) $\mathcal{E} \in \operatorname{CPTP}(A \rightarrow X)$. The quantity $\underline{\mathrm{D}}$ is usually known as the measured relative entropy [10]. The equality in Eq. (12) means that any quantum divergence that reduces to the classical KL relative entropy must be no smaller than the Umegaki relative entropy (for more details on optimal extensions, see Ref. [5]). In Ref. [10], it has been shown that Eq. (12) also holds if $D$ is replaced with the sandwiched or minimal quantum Rényi divergence of order $\alpha \in[1 / 2, \infty][8,9]$.

Recently, the extensions of quantum divergences to channel divergences have been studied intensively [14-19]. Examples include the channel extension of the Umegaki relative entropy, given for all $\mathcal{N}, \mathcal{M} \in$ $\operatorname{CPTP}(A \rightarrow B)$ by

$$
D(\mathcal{M} \| \mathcal{N}):=\sup _{\rho \in \mathfrak{D}(R A)} D\left(\mathcal{M}^{A \rightarrow B}\left(\rho^{R A}\right) \| \mathcal{N}^{A \rightarrow B}\left(\rho^{R A}\right),\right.
$$

where the supremum is over all systems $R$ and all density matrices in $\mathfrak{D}(R A)$. It can be shown [14] that the above supremum can be replaced with a maximum over all pure states in $\mathfrak{D}(R A)$ with $|R|=|A|$. The min and max relative entropies have also been extended to quantum channels. They are given for all $\mathcal{N}, \mathcal{M} \in \operatorname{CPTP}(A \rightarrow B)$ by

$$
D_{\max }(\mathcal{M} \| \mathcal{N}):=\log \min \{t \geq 0: t \mathcal{N} \geq \mathcal{M}\}
$$

(recall that $t \mathcal{N} \geq \mathcal{M}$ means that $t \mathcal{N}-\mathcal{M}$ is a CP map) if $\operatorname{supp}\left(J_{\mathcal{N}}\right) \subseteq \operatorname{supp}\left(J_{\mathcal{M}}\right)$ and $D_{\max }(\mathcal{M} \| \mathcal{N})=\infty$ otherwise and

$$
D_{\min }(\mathcal{M} \| \mathcal{N}):=\max _{\psi \in \mathfrak{D}(R A)}\left\{-\log \operatorname{Tr}\left[\mathcal{N}(\psi) \Pi_{\mathcal{M}(\psi)}\right]\right\}
$$

if for all $\psi \in \mathfrak{D}(R A), \operatorname{Tr}[\mathcal{N}(\psi) \mathcal{M}(\psi)] \neq 0$, and $D_{\text {min }}$ $(\mathcal{M} \| \mathcal{N})=\infty$ otherwise. We assume above that $|R|=|A|$. The divergence $D_{\min }(\mathcal{M} \| \mathcal{N})$ can be viewed as the $\epsilon=0$ case of the hypothesis-testing channel divergence given by

$D_{\min }^{\epsilon}(\mathcal{M} \| \mathcal{N}):=\max _{\psi \in \mathcal{D}(R A)} D_{\min }^{\epsilon}\left[\mathcal{M}^{A \rightarrow B}\left(\psi^{R A}\right) \| \mathcal{N}^{A \rightarrow B}\left(\psi^{R A}\right)\right]$,

where on the left-hand side, $D_{\min }^{\varepsilon}$ corresponds to a channel divergence, whereas on the right-hand side, $D_{\min }^{\varepsilon}$ corresponds to a state divergence. We can assume above that
$|R|=|A|$, the maximum is over all pure states in $\mathfrak{D}(R A)$, and for any $\rho, \sigma \in \mathfrak{D}(A)$. We discuss more examples of channel divergences later on.

\section{Relative majorization}

We say that a pair of vectors $\mathbf{p}, \mathbf{q} \in \mathfrak{D}(X)$ is relatively majorized by another pair of vectors $\mathbf{p}^{\prime}, \mathbf{q}^{\prime} \in \mathfrak{D}(Y)$ and write

$$
(\mathbf{p}, \mathbf{q}) \succ\left(\mathbf{p}^{\prime}, \mathbf{q}^{\prime}\right)
$$

if there exists a stochastic evolution matrix $\mathcal{E} \in$ $\operatorname{CPTP}(X \rightarrow Y)$ such that $\left(\mathbf{p}^{\prime}, \mathbf{q}^{\prime}\right)=[\mathcal{E}(\mathbf{p}), \mathcal{E}(\mathbf{q})]$. Therefore, the relative Rényi entropies behave monotonically under relative majorization; i.e., if $(\mathbf{p}, \mathbf{q}) \succ\left(\mathbf{p}^{\prime}, \mathbf{q}^{\prime}\right)$, then $D_{\alpha}(\mathbf{p} \| \mathbf{q}) \geq D_{\alpha}\left(\mathbf{p}^{\prime} \| \mathbf{q}^{\prime}\right)$ for all $\alpha \in[0, \infty]$. If we have $(\mathbf{p}, \mathbf{q}) \succ\left(\mathbf{p}^{\prime}, \mathbf{q}^{\prime}\right) \succ(\mathbf{p}, \mathbf{q})$, then we write

$$
\left(\mathbf{p}^{\prime}, \mathbf{q}^{\prime}\right) \sim(\mathbf{p}, \mathbf{q}) .
$$

Relative majorization is a partial order that can be characterized with testing regions. The testing region of a pair of probability vectors $\mathbf{p}, \mathbf{q} \in \mathcal{D}(n)$ is a region in $\mathbb{R}^{2}$ defined as

$\mathfrak{T}(\mathbf{p}, \mathbf{q}):=\left\{(\mathbf{p} \cdot \mathbf{t}, \mathbf{q} \cdot \mathbf{t}) \in \mathbb{R}^{2}: 0 \leq \mathbf{t} \leq(1, \ldots, 1)^{T}, \mathbf{t} \in \mathbb{R}^{n}\right\}$

where the inequalities are entry-wise. This region is bounded by two curves known as the lower and upper Lorenz curves.

The upper Lorenz curve can be obtained from the lower Lorenz curve by a rotation of $180^{\circ}$ around the point $\left(\frac{1}{2}, \frac{1}{2}\right) \in \mathbb{R}^{2}$. Therefore, the lower (or upper) Lorenz curve uniquely determines the testing region. The lower Lorenz curve of a pair of probability vectors $\mathbf{p}, \mathbf{q} \in \mathbb{R}^{n}$, denoted here by $\mathcal{L}(\mathbf{p}, \mathbf{q})$, has $n+1$ vertices that can be computed as follows. First, observe that the testing region is invariant under the transformation $(\mathbf{p}, \mathbf{q}) \rightarrow(\Pi \mathbf{p}, \Pi \mathbf{q})$, where $\Pi$ is any permutation matrix. Therefore, without loss of generality, we can assume that the components of $\mathbf{p}$ and $\mathbf{q}$ are arranged such that

$$
\frac{p_{1}}{q_{1}} \geq \frac{p_{2}}{q_{2}} \geq \cdots \geq \frac{p_{n}}{q_{n}} .
$$

The $n+1$ vertices of $\mathcal{L}(\mathbf{p}, \mathbf{q})$ are the $(0,0)$ vertex and the $n$ vertices $\left\{\left(a_{\ell}, b_{\ell}\right)\right\}_{\ell=1}^{n} \subset \mathcal{L}(\mathbf{p}, \mathbf{q})$, where

$$
a_{\ell}=\sum_{x=1}^{\ell} p_{x} \quad \text { and } \quad b_{\ell}=\sum_{x=1}^{\ell} q_{x} .
$$

The relevance of testing regions to our study here is the following theorem that goes back to Blackwell [38] and that since then has been rediscovered under different 
names including $d$ majorization [39], matrix majorization [40], and thermomajorization [41] (see also the book on majorization by Marshall and Olkin [42]).

Theorem (see Ref. [38]). Let $\mathbf{p}, \mathbf{q} \in \mathcal{P}(n)$ and $\mathbf{p}^{\prime}, \mathbf{q}^{\prime} \in$ $\mathcal{P}(m)$ be two pairs of probability vectors in dimensions $n$ and $m$, respectively. Then,

$$
(\mathbf{p}, \mathbf{q}) \succ\left(\mathbf{p}^{\prime}, \mathbf{q}^{\prime}\right) \quad \Longleftrightarrow \mathfrak{T}(\mathbf{p}, \mathbf{q}) \supseteq \mathfrak{T}\left(\mathbf{p}^{\prime}, \mathbf{q}^{\prime}\right) .
$$

The above theorem provides a geometric characterization to relative majorization, that is, $(\mathbf{p}, \mathbf{q}) \succ\left(\mathbf{p}^{\prime}, \mathbf{q}^{\prime}\right)$ if and only if the lower Lorenz curve of $\left(\mathbf{p}^{\prime}, \mathbf{q}^{\prime}\right)$ is nowhere below the lower Lorenz curve of $(\mathbf{p}, \mathbf{q})$. Relative majorization has another remarkable property that has been proven in Ref. [4].

Theorem (see Ref. [4]). Let $\mathbf{p}, \mathbf{q} \in \mathfrak{D}(X)$ and set $a:=$ $|X|$. Suppose that the components of $\mathbf{q}$ are positive and rational. That is, there exists $n_{1}, \ldots, n_{a} \in \mathbb{N}$ such that

$$
\mathbf{q}=\left(\frac{n_{1}}{n}, \ldots, \frac{n_{a}}{n}\right), \quad n:=\sum_{x=1}^{a} n_{x} .
$$

Let

$$
\mathbf{r}:=\bigoplus_{x=1}^{a} p_{x} \mathbf{u}^{\left(n_{x}\right)} \in \mathfrak{D}(n),
$$

where each $\mathbf{u}^{\left(n_{x}\right)}$ is an $n_{x}$-dimensional uniform distribution. Then,

$$
(\mathbf{p}, \mathbf{q}) \sim(\mathbf{r}, \mathbf{u})
$$

where $\mathbf{u} \in \mathfrak{D}(n)$ is the n-dimensional uniform distribution.

\section{CHANNEL DIVERGENCES}

Quantum divergences have numerous applications in quantum-information theory [3] and quantum resource theories [35]. As discussed in Sec. II, their defining property is the DPI. Therefore, to define a channel divergence, one has to extend the DPI to the channel domain. Since the most general and physically realizable operation that can be applied to a quantum channel is a superchannel, we define channel divergences in terms of superchannels.

We start with the formal definition of a channel divergence and a channel relative entropy. We use the notation $\mathbb{D}$ for a general channel divergence, to distinguish it from a general quantum divergence $\mathbf{D}$ or a general classical divergence $\mathscr{D}$.

\section{Definition 1: Let}

$$
\mathbb{D}: \bigcup_{A, B}\{\operatorname{CPTP}(A \rightarrow B) \times \operatorname{CPTP}(A \rightarrow B)\} \rightarrow \mathbb{R} \cup\{\infty\}
$$

be a function acting on pairs of quantum channels in finite dimensions.
1. The function $\mathbb{D}$ is called a channel divergence if it satisfies the generalized DPI. That is, for any $\mathcal{M}, \mathcal{N} \in$ $\mathrm{CPTP}(A \rightarrow B)$ and a superchannel $\Theta \in \mathfrak{S}\left(A B \rightarrow A^{\prime} B^{\prime}\right)$

$$
\mathbb{D}(\Theta[\mathcal{M}] \| \Theta[\mathcal{N}]) \leq \mathbb{D}(\mathcal{M} \| \mathcal{N}) .
$$

2. A channel divergence $\mathbb{D}$ is called a channel relative entropy if it satisfies the following additional properties:

(a) Additivity. For any $\mathcal{M}_{1}, \mathcal{M}_{2} \in \operatorname{CPTP}(A \rightarrow B)$ and any $\mathcal{N}_{1}, \mathcal{N}_{2} \in \operatorname{CPTP}\left(A^{\prime} \rightarrow B^{\prime}\right)$,

$\mathbb{D}\left(\mathcal{M}_{1} \otimes \mathcal{M}_{2} \| \mathcal{N}_{1} \otimes \mathcal{N}_{2}\right)=\mathbb{D}\left(\mathcal{M}_{1} \| \mathcal{N}_{1}\right)+\mathbb{D}\left(\mathcal{M}_{2} \| \mathcal{N}_{2}\right)$

(b) Normalization.

$$
\mathbb{D}\left(\left[\begin{array}{ll}
1 & 0 \\
0 & 0
\end{array}\right] \|\left[\begin{array}{cc}
1 / 2 & 0 \\
0 & 1 / 2
\end{array}\right]\right)=1,
$$

where quantum states are viewed as channels with onedimensional input.

Remark.-The term "channel relative entropy" is sometimes used in the literature to refer to a quantity that extends the Umegaki relative entropy as in Eq. (63). However, in Ref. [29], it has been shown not to be additive under tensor products and therefore it does not fit with our above definition of a relative entropy. Moreover, its regularized version, which has been shown in Ref. [29] to be equal to the amortized divergence (and which is known to be additive [43]), is the one that appears in most applications. We therefore choose to call the expression in Eq. (63) a channel divergence rather than a channel relative entropy.

As an example, consider $D_{\max }(\mathcal{M} \| \mathcal{N})$ as defined in Eq. (15). This channel divergence has several operational interpretations in quantum information [18-29]. One can easily verify that it is additive and satisfies the normalization condition of a relative entropy. It also satisfies the DPI as defined above. This can be seen from the fact that if $t \mathcal{N}-\mathcal{M}$ is a CP map, then $\Theta[\mathcal{N}-t \mathcal{M}]=\Theta[\mathcal{N}]-t \Theta[\mathcal{M}]$ is also a CP map, so that $D_{\max }(\Theta[\mathcal{M}] \| \Theta[\mathcal{N}]) \leq D_{\max }(\mathcal{M} \| \mathcal{N})$. Therefore, according to the above definition, $D_{\max }(\mathcal{M} \| \mathcal{N})$ is a channel relative entropy.

Another important example is the hypothesis-testing divergence $D_{\min }^{\epsilon}(\mathcal{M} \| \mathcal{N})$ as defined in Eq. (17). This divergence plays a key role in hypothesis testing with quantum channels [25], although it is not additive (unless $\varepsilon=0$ ), which means that it is not a relative entropy according to our definition. Still, it satisfies the DPI for channels [17].

Also, the diamond norm is a channel divergence according to our above definition, since it satisfies the channel DPI [17] and it has an operational interpretation in terms of the optimal success probability of distinguishing between two channels (see, e.g., Ref. [44]). This again supports our definition of channel divergences, particularly the extension of the DPI with superchannels. This channel version 
of the DPI is satisfied by channel divergences that have physical and operational interpretations. We discuss more examples of such channel divergences with operational interpretation in the concluding section.

Channel divergences can be viewed as a generalization of quantum divergences, since the $\operatorname{set} \operatorname{CPTP}(A \rightarrow B)$ with $|A|=1$ can be viewed as the set of quantum states $\mathfrak{D}(B)$. Hence, we view here the quantum states in $\mathfrak{D}(B)$, i.e., those in $\mathrm{CPTP}(1 \rightarrow B)$, where 1 represents the onedimensional trivial system, as a special type of quantum channel. However, there is another type of quantum channel that can be identified with quantum states. This is the replacement channel. Let $\sigma \in \mathfrak{D}(B)$ and define the channel $\mathcal{R}_{\sigma} \in \operatorname{CPTP}(A \rightarrow B)$ with $|A|>1$ as

$$
\mathcal{R}_{\sigma}\left(\omega^{A}\right):=\operatorname{Tr}\left[\omega^{A}\right] \sigma^{B} \quad \forall \omega \in \mathfrak{L}(A) .
$$

Therefore, this channel is uniquely determined by the dimension of system $A$ and the state $\sigma^{B}$. It is therefore natural to ask if channel divergences between replacement channels reduce to quantum divergences between the states that define the replacement channels. Not too surprisingly, we see below that the answer to this question is in the affirmative.

\section{A. Basic properties}

Channel divergences and relative entropies borrow some of their properties from quantum divergences. In this section, we discuss a few of these basic properties. The significance of these properties is that they are satisfied by all channel divergences (or relative entropies) and therefore are not unique to a particular divergence. Later on, we will see that some divergences have additional unique properties that are not satisfied by all divergences. We say that a channel divergence $\mathbb{D}$ is faithful if $\mathbb{D}(\mathcal{N} \| \mathcal{M})=0$ implies that $\mathcal{M}=\mathcal{N}$.

Theorem 1 (Properties of Channel Divergences). Let $\mathbb{D}$ be a channel divergence. Then,

1. If $\mathbb{D}(1 \| 1)=0$ [here, 1 stands for the trivial channel in $C P T P(1 \rightarrow 1)]$, then for any two channels $\mathcal{M}, \mathcal{N} \in$ $C P T P(A \rightarrow B)$,

$$
\mathbb{D}(\mathcal{M} \| \mathcal{N}) \geq 0,
$$

with equality if $\mathcal{M}=\mathcal{N}$.

2. If $\mathbb{D}$ is a channel relative entropy, then $\mathbb{D}(1 \| 1)=0$.

3. $\mathbb{D}$ is faithful if and only if its reduction to classical states (i.e., probability vectors) is faithful.

4. For any two replacement channels, $\mathcal{R}_{\sigma_{1}}, \mathcal{R}_{\sigma_{2}} \in$ $C P T P(A \rightarrow B)$, as defined in Eq. (27) with $\sigma_{1}, \sigma_{2} \in \mathfrak{D}(B)$ and $|A|>1$

$$
\mathbb{D}\left(\mathcal{R}_{\sigma_{1}} \| \mathcal{R}_{\sigma_{2}}\right)=\mathbb{D}\left(\sigma_{1} \| \sigma_{2}\right)
$$

5. Suppose that $\mathbb{D}$ is a channel relative entropy and let $\mathcal{E}_{1}, \ldots, \mathcal{E}_{n} \in \operatorname{CPTP}(A \rightarrow B)$ be a set of $n$ orthogonal quantum channels; i.e., their Choi matrices satisfy $\operatorname{Tr}\left[J_{\mathcal{E}_{j}}^{A B} J_{\mathcal{E}_{k}}^{A B}\right]=0$ for all $j \neq k \in[n]$. Then, for any probability vector $\mathbf{p}=\left\{p_{x}\right\}_{x=1}^{n}$ and $\mathcal{N}:=\sum_{x=1}^{n} p_{x} \mathcal{E}_{x}$,

$$
\mathbb{D}\left(\mathcal{E}_{x} \| \mathcal{N}\right)=-\log \left(p_{x}\right) \quad \forall x=1, \ldots, n .
$$

6. If $\mathbb{D}$ is a channel relative entropy, then for all $\mathcal{M}, \mathcal{N} \in \operatorname{CPTP}(A \rightarrow B)$

$$
D_{\min }(\mathcal{M} \| \mathcal{N}) \leq \mathbb{D}(\mathcal{M} \| \mathcal{N}) \leq D_{\max }(\mathcal{M} \| \mathcal{N}),
$$

where $D_{\min }$ and $D_{\max }$ are the channel min and max relative entropies as defined in Eqs. (17) and (15), respectively.

7. Let $\mathbb{D}$ be a channel relative entropy, let $\mathcal{R} \in$ $\operatorname{CPTP}(A \rightarrow B)$ be the completely randomizing channel (i.e., $\mathcal{R}=\mathcal{R}_{\mathbf{u}^{B}}$, meaning that $\mathcal{R}\left(\rho^{A}\right)=\mathbf{u}^{B}$ is the maximally mixed state $\mathbf{u}^{B}$ for all $\left.\rho \in \mathfrak{D}(A)\right)$, and let $\mathcal{V} \in$ $C P T P(A \rightarrow B)$ be an isometry channel (we assume $|A| \leq$ $|B|)$. Then,

$$
\mathbb{D}\left(\mathcal{V}^{A \rightarrow B} \| \mathcal{R}^{A \rightarrow B}\right)=\log |A B| .
$$

8. If $\mathbb{D}$ is a channel relative entropy, then for any $\mathcal{N}, \mathcal{M}, \mathcal{E} \in \operatorname{CPTP}(A \rightarrow B)$,

$$
\mathbb{D}(\mathcal{N} \| \mathcal{M}) \leq \mathbb{D}(\mathcal{N} \| \mathcal{E})+D_{\max }(\mathcal{E} \| \mathcal{M})
$$

9. If $\mathbb{D}$ is a channel relative entropy, then for any $\mathcal{N}, \mathcal{E}, \mathcal{M} \in \operatorname{CPTP}(A \rightarrow B)$,

$$
\begin{aligned}
& \mathbb{D}(\mathcal{N} \| \mathcal{M})-\mathbb{D}(\mathcal{E} \| \mathcal{M}) \\
& \quad \leq \min _{0 \leq s \leq 2^{-D} \max (\mathcal{E} \| \mathcal{N})} D_{\max }(\mathcal{N}+s(\mathcal{M}-\mathcal{E}) \| \mathcal{M}) .
\end{aligned}
$$

Moreover, if $J_{\mathcal{N}}, J_{\mathcal{E}}$, and $J_{\mathcal{M}}$ have full support, then

$$
\mathbb{D}(\mathcal{N} \| \mathcal{M})-\mathbb{D}(\mathcal{E} \| \mathcal{M}) \leq \log \left(1+\frac{\left\|J_{\mathcal{N}}-J_{\mathcal{E}}\right\|_{\infty}}{\lambda_{\min }\left(J_{\mathcal{E}}\right) \lambda_{\min }\left(J_{\mathcal{M}}\right)}\right)
$$

Remark.-Property 8 implies that for any $\mathcal{N}, \mathcal{E}, \mathcal{F} \in$ $\mathrm{CPTP}(A \rightarrow B)$,

$$
|\mathbb{D}(\mathcal{N} \| \mathcal{E})-\mathbb{D}(\mathcal{N} \| \mathcal{F})| \leq D_{T}(\mathcal{E} \| \mathcal{F}),
$$

where

$$
D_{T}(\mathcal{E} \| \mathcal{F}):=\max \left\{D_{\max }(\mathcal{E} \| \mathcal{F}), D_{\max }(\mathcal{F} \| \mathcal{E})\right\}
$$

is a metric (the state version of which is known as the Thompson metric) on $\operatorname{CPTP}(A \rightarrow B)$. Hence, in particular, any channel relative entropy $\mathbb{D}$ is continuous in its 
second argument on the subset of $\operatorname{CPTP}(A \rightarrow B)$ consisting of channels that have strictly positive Choi matrices. Similarly, Property 9 implies a continuity in the first argument of $\mathbb{D}$. That is, if $\mathcal{E}$ is very close to $\mathcal{N}$, then $s$ can be taken to be very close to one as long as $\operatorname{supp}\left(J_{\mathcal{E}}\right) \subseteq \operatorname{supp}\left(J_{\mathcal{N}}\right)$. Note that in this case, if we also have $\operatorname{supp}\left(J_{\mathcal{N}}\right) \subseteq \operatorname{supp}\left(J_{\mathcal{M}}\right)$, then the continuity of $D_{\max }$ implies that $D_{\max }(\mathcal{N}+s(\mathcal{M}-\mathcal{E}) \| \mathcal{M})$ goes to zero as $s$ goes to one (recall that if $s=1$, then $\mathcal{E}=\mathcal{N}$ ).

\section{B. Divergences of classical channels}

The properties discussed in the previous subsection apply to all quantum-channel divergences. Here, we show that there are additional properties satisfied by channel relative entropies, when the inputs are restricted to be classical. That is, in this subsection we consider classical dynamical divergences; i.e., divergences of classical channels. Most interestingly, we see that there is only one classical-channel relative entropy that reduces to the KL divergence. We start with the following theorem.

Theorem 2. Let $\mathbb{D}$ be a classical channel divergence that reduces to the classical (state) divergence $\mathscr{D}$ on classical states in $\mathfrak{D}(X) \times \mathcal{D}(X)$. Suppose further that $\mathscr{D}$ is quasiconvex. Then, for all classical channels $\mathcal{M}, \mathcal{N} \in$ $C P T P(X \rightarrow Y)$,

$$
\underline{\mathscr{D}}(\mathcal{M} \| \mathcal{N}) \leq \mathbb{D}(\mathcal{M} \| \mathcal{N}) \leq \overline{\mathscr{D}}(\mathcal{M} \| \mathcal{N}),
$$

where

$$
\begin{aligned}
& \underline{\mathscr{D}}(\mathcal{M} \| \mathcal{N}):=\max _{x \in\{1, \ldots,|X|\}} \mathscr{D}(\mathcal{M}(|x\rangle\langle x|) \| \mathcal{N}(|x\rangle\langle x|)), \\
& \overline{\mathscr{D}}(\mathcal{M} \| \mathcal{N}):=\inf _{|Z| \in \mathbb{N}} \mathscr{D}(\mathbf{p} \| \mathbf{q}),
\end{aligned}
$$

where the infimum is over all $\mathbf{p}, \mathbf{q} \in \mathfrak{D}(Z)$ that satisfy $(\mathbf{p}, \mathbf{q}) \succ[\mathcal{M}(|x\rangle\langle x|), \mathcal{N}(|x\rangle\langle x|)]$ for all $x \in[|X|]$. Moreover, $\mathscr{D}$ is a classical channel relative entropy and $\overline{\mathscr{D}}$ is a normalized classical channel divergence.

Remark.-In the next section, we develop a general framework to extend channel divergences from one domain to a larger one and the optimality of the divergences $\mathscr{D}$ and $\overline{\mathscr{D}}$ follows trivially from that general formalism. Hence, the theorem above can be viewed as a corollary of the third property in Theorem 5 of the next section. Moreover, we see shortly, from the closed formula for $\overline{\mathscr{D}}(\mathcal{M} \| \mathcal{N})$, that, without loss of generality, we can bound $|Z| \leq|Y|$.

To illustrate the above expression for $\overline{\mathscr{D}}$, in Fig. 2 we draw three lower Lorenz curves associated with the two channels $\mathcal{N}, \mathcal{M} \in \operatorname{CPTP}(X \rightarrow Y)$, where $|X|=$ 2 and $|Y|=4$. In this example, the channel $\mathcal{M}$ is defined via the probability vectors $\mathbf{m}_{0}:=\mathcal{M}(|0\rangle\langle 0|)=$

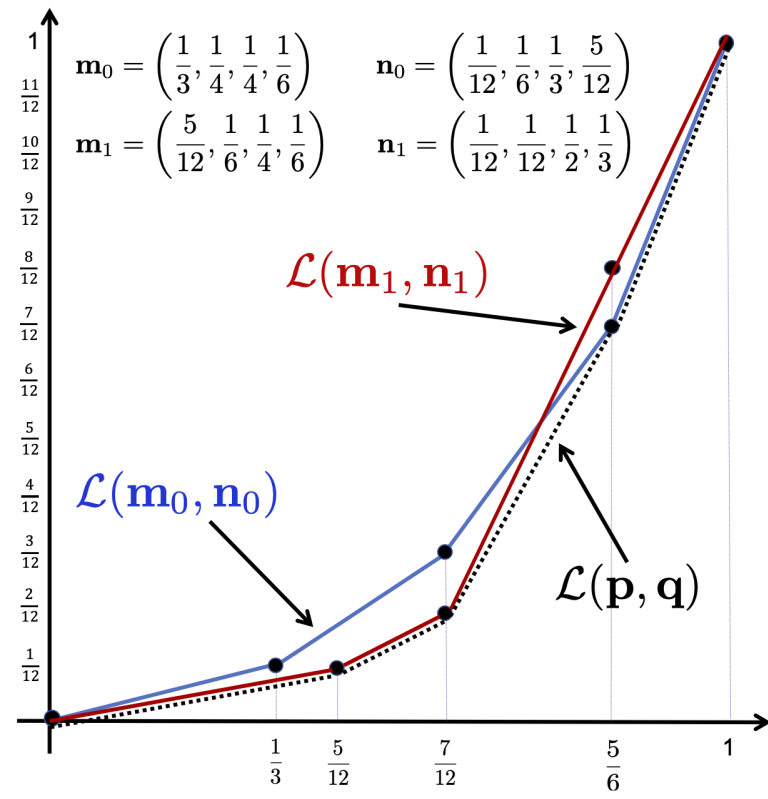

FIG. 2. Three lower Lorenz curves. The channels $\mathcal{M}, \mathcal{N} \in$ $\operatorname{CPTP}(X \rightarrow Y)$ with $|X|=2$ and $|Y|=4$.

$(1 / 3,1 / 4,1 / 4,1 / 6)$ and $\mathbf{m}_{1}:=\mathcal{M}(|1\rangle\langle 1|)=(5 / 12,1 / 6$, $1 / 4,1 / 6)$ and the channel $\mathcal{N}$ via $\mathbf{n}_{0}:=\mathcal{N}(|0\rangle\langle 0|)=$ $(1 / 12,1 / 6,1 / 3,5 / 12)$ and $\mathbf{n}_{1}:=\mathcal{N}(|1\rangle\langle 1|)=(1 / 12,1 / 12$, $1 / 2,1 / 3)$. The figure helps to see how to compute the optimal pair $(\mathbf{p}, \mathbf{q})$ that satisfies both $(\mathbf{p}, \mathbf{q}) \succ\left(\mathbf{m}_{0}, \mathbf{n}_{0}\right)$ and $(\mathbf{p}, \mathbf{q}) \succ\left(\mathbf{m}_{1}, \mathbf{n}_{1}\right)$. The Lorenz curve, $\mathcal{L}(\mathbf{p}, \mathbf{q})$, must be the closest possible curve to both $\mathcal{L}\left(\mathbf{m}_{0}, \mathbf{n}_{0}\right)$ and $\mathcal{L}\left(\mathbf{m}_{1}, \mathbf{n}_{1}\right)$ and it must also be below both of them. In Fig. 2, the dashed line is the Lorenz curve of $\mathcal{L}(\mathbf{p}, \mathbf{q})$. Its vertices are $(0,0),(5 / 12,1 / 12),(7 / 12,2 / 12),(5 / 6,7 / 12)$, and $(1,1)$. This reveals that the optimal $\mathbf{p}$ and $\mathbf{q}$ are given by $\mathbf{p}=$ $(5 / 12,1 / 6,1 / 4,1 / 6)$ and $\mathbf{q}=(1 / 12,1 / 12,5 / 12,5 / 12)$.

For the general case, we provide in the theorem below a closed formula for $\overline{\mathscr{D}}(\mathcal{M} \| \mathcal{N})$ for any two classical channels $\mathcal{M}, \mathcal{N} \in \operatorname{CPTP}(X \rightarrow Y)$ in finite dimensions $|X|,|Y|<\infty$. For any $x=1, \ldots,|X|$, we denote by $\left(\mathbf{m}_{x}, \mathbf{n}_{x}\right):=[\mathcal{M}(|x\rangle\langle x|), \mathcal{N}(|x\rangle\langle x|)]$ and by $M=\left(M_{y \mid x}\right)$ and $N=\left(N_{y \mid x}\right)$ the $|Y| \times|X|$ matrices the components of which are $M_{y \mid x}=\left\langle y\left|\mathbf{m}_{x}\right| y\right\rangle$ and $N_{y \mid x}=\left\langle y\left|\mathbf{n}_{x}\right| y\right\rangle$, respectively. We also rearrange the components of the columns of $M$ and $N$ such that for each $x=1, \ldots,|X|$

$$
\frac{M_{1 \mid x}}{N_{1 \mid x}} \geq \frac{M_{2 \mid x}}{N_{2 \mid x}} \geq \cdots \geq \frac{M_{|Y| \mid x}}{N_{|Y| \mid x}} .
$$

Therefore, aside from the points $(0,0)$ and $(1,1)$, for any $x=1, \ldots,|X|$, the vertices of the lower Lorenz curve of $\mathcal{L}\left(\mathbf{m}_{x}, \mathbf{n}_{x}\right)$ are given by $\left\{\left(a_{z x}, b_{z x}\right)\right\}_{z=1}^{|Y|-1}$, where

$$
a_{z x}:=\sum_{y=1}^{z} M_{y \mid x} \quad \text { and } \quad b_{z x}:=\sum_{y=1}^{z} N_{y \mid x} .
$$


Theorem 3 (Closed Formula). Let $\mathbf{D}$ be a classical divergences and let $\mathcal{N}, \mathcal{M} \in C P T P(X \rightarrow Y)$. Using the above notation, the maximal classical channel extension $\overline{\mathscr{D}}$ is given by

$$
\overline{\mathscr{D}}(\mathcal{M} \| \mathcal{N})=\mathscr{D}(\mathbf{p} \| \mathbf{q})
$$

where $\mathbf{p}=\left\{p_{z}\right\}_{z=1}^{|Y|}$ and $\mathbf{q}=\left\{q_{z}\right\}_{z=1}^{|Y|}$ are $|Y|$-dimensional probability vectors given by

$$
\begin{aligned}
& p_{1}=M_{1 x_{1}}, \quad p_{z}=a_{z x_{z}}-a_{(z-1) x_{z-1}} \\
& q_{1}=N_{1 x_{1}}, \quad q_{z}=b_{z x_{z}}-b_{(z-1) x_{z-1}} \quad \forall z=2, \ldots,|Y|
\end{aligned}
$$

where $x_{1}, \ldots, x_{|Y|} \in\{1, \ldots,|X|\}$ are defined by induction via the relations

$$
\frac{N_{1 x_{1}}}{M_{1 x_{1}}}=\min _{x \in[|X|]} \frac{N_{1 x}}{M_{1 x}}
$$

and for any $z=2, \ldots,|Y|$,

$$
\frac{b_{z x_{z}}-b_{(z-1) x_{z-1}}}{a_{z x_{z}}-a_{(z-1) x_{z-1}}}=\min _{x \in\{1, \ldots,|X|\}} \frac{b_{z x}-b_{(z-1) x_{z-1}}}{a_{z x}-a_{(z-1) x_{z-1}}} .
$$

The maximal divergence $\overline{\mathscr{D}}$ is in general not additive and, therefore, is not a relative entropy. In the next result, we prove that if $\mathbb{D}$ is a channel relative entropy that reduces to the $\mathrm{KL}$ relative entropy, then it is unique.

\section{Uniqueness of the Channel KL Relative Entropy}

Theorem 4. Let $\mathbb{D}$ be a classical channel divergence that reduces to the Kullback-Leibler divergence, D, on classical states. If $\mathbb{D}$ is continuous in its second argument, then for all $\mathcal{N}, \mathcal{M} \in C P T P(X \rightarrow Y)$,

$$
\begin{aligned}
& \mathbb{D}(\mathcal{M} \| \mathcal{N})=\max _{x \in\{1, \ldots,|X|\}} \\
& D[\mathcal{M}(|x\rangle\langle x|) \| \mathcal{N}(|x\rangle\langle x|)] .
\end{aligned}
$$

The above uniqueness theorem holds only for the KL divergence and it is not clear whether this uniqueness still holds for the case in which the KL divergence is replaced with the Rényi divergences. In Ref. [5] (cf. Refs. [36,37]), it has been shown that the Umegaki relative entropy (and, in particular, the KL divergence) is the only asymptotically continuous divergence. Therefore, the above uniqueness theorem implies that there is only one classical channel divergence that is asymptotically continuous on classical states.

\section{OPTIMAL EXTENSIONS}

In this section, we apply the extension techniques developed in Ref. [5] for general resource theories to study the optimal extensions of a classical divergence to a channel divergence. We point out that the extensions that we consider are optimal in the sense that they are either minimal or maximal among all possible extensions. For example, if $\mathbb{D}(\mathcal{N} \| \mathcal{M})$ is a channel divergence that reduces to a classical state divergence $\mathscr{D}(\mathbf{p} \| \mathbf{q})$ when $\mathcal{N}=\mathbf{p}$ and $\mathcal{M}=\mathbf{q}$ are classical states, then $\mathbb{D}(\mathcal{N} \| \mathcal{M})$ is bounded from below by the minimal extension of $\mathscr{D}(\mathbf{p} \| \mathbf{q})$ to the channel domain and bounded from above by the maximal extension of $\mathscr{D}(\mathbf{p} \| \mathbf{q})$ to the channel domain. One can also consider extensions of quantum divergences to channel divergences. As we will see, such extensions give rise to additional types of channel divergences. We start with the general framework for channel extensions of divergences.

\section{A. General framework for extensions}

In the following theorem, we apply the results that have been given in Ref. [5] for a general resource theory to channel extensions of divergences. We start with the definition of an $\Re$ divergence.

Definition 2: Let $\mathfrak{R}(A \rightarrow B) \subset \operatorname{CPTP}(A \rightarrow B)$ be a subset of quantum channels for any two physical systems $A$ and $B$. A function

$$
\mathbf{C}: \bigcup_{A, B} \mathfrak{R}(A \rightarrow B) \times \mathfrak{R}(A \rightarrow B) \rightarrow \mathbb{R}_{+}
$$

is called an $\mathfrak{R}$ divergence if for any $\mathcal{M}, \mathcal{N} \in \mathfrak{R}(A \rightarrow$ $B)$ and any superchannel $\Theta \in \mathfrak{S}\left(A B \rightarrow A^{\prime} B^{\prime}\right)$ such that $\Theta[\mathcal{M}] \in \mathfrak{R}\left(A^{\prime} \rightarrow B^{\prime}\right)$ and $\Theta[\mathcal{N}] \in \mathfrak{R}\left(A^{\prime} \rightarrow B^{\prime}\right)$,

$$
\mathbf{C}(\Theta[\mathcal{M}] \| \Theta[\mathcal{N}]) \leq \mathbf{C}(\mathcal{M} \| \mathcal{N}) .
$$

Any $\mathfrak{R}$ divergence has two optimal extensions to a quantum channel divergence:

1. The minimal channel extension, for any $\mathcal{M}, \mathcal{N} \in$ $\operatorname{CPTP}(A \rightarrow B)$ :

$$
\underline{\mathbf{C}}(\mathcal{M} \| \mathcal{N}):=\sup \mathbf{C}(\Theta[\mathcal{M}] \| \Theta[\mathcal{N}]),
$$

where the supremum is over all systems $A^{\prime}, B^{\prime}$ and all $\Theta \in$ $\mathfrak{S}\left(A B \rightarrow A^{\prime} B^{\prime}\right)$ such that $\Theta[\mathcal{M}], \Theta[\mathcal{N}] \in \mathfrak{R}\left(A^{\prime} \rightarrow B^{\prime}\right)$.

2. The maximal channel extension, for any $\mathcal{M}, \mathcal{N} \in$ $\operatorname{CPTP}(A \rightarrow B)$ :

$$
\overline{\mathbf{C}}(\mathcal{M} \| \mathcal{N}):=\inf \mathbf{C}(\mathcal{E} \| \mathcal{F}),
$$

where the infimum is also over all systems $A^{\prime}, B^{\prime}$ and all $\mathcal{E}, \mathcal{F} \in \mathfrak{R}\left(A^{\prime} \rightarrow B^{\prime}\right)$ such that there exists $\Theta \in \mathfrak{S}\left(A^{\prime} B^{\prime} \rightarrow\right.$ $A B$ ) that satisfies $\mathcal{M}=\Theta[\mathcal{E}]$ and $\mathcal{N}=\Theta[\mathcal{F}]$. 
Remark.-In the above definition, we assume that $\mathfrak{R}(A \rightarrow$ $B$ ) is a subset of quantum channels. By taking $\mathfrak{R}(A \rightarrow B)$ to be a subset of replacement channels as in Eq. (27), the above extensions can also be applied to state divergences.

Theorem 5. Let $\mathfrak{R}(A \rightarrow B) \subset C P T P(A \rightarrow B)$, and let $\mathbf{C}$ be an $\mathfrak{R}$ divergence. Then, its maximal and minimal channel extensions $\bar{C}$ and $\underline{C}$ have the following properties :

1. Reduction. For any $\mathcal{M}, \mathcal{N} \in \mathfrak{R}(A \rightarrow B)$,

$$
\underline{\mathbf{C}}(\mathcal{M} \| \mathcal{N})=\overline{\mathbf{C}}(\mathcal{M} \| \mathcal{N})=\mathbf{C}(\mathcal{M} \| \mathcal{N}) .
$$

2. Data Processing Inequality. For any $\mathcal{M}, \mathcal{N} \in$ $C P T P(A \rightarrow B)$ and any $\Theta \in \mathfrak{S}\left(A B \rightarrow A^{\prime} B^{\prime}\right)$,

$$
\begin{aligned}
& \underline{\mathbf{C}}(\Theta[\mathcal{M}] \| \Theta[\mathcal{N}]) \leq \underline{\mathbf{C}}(\mathcal{M} \| \mathcal{N}), \\
& \overline{\mathbf{C}}(\Theta[\mathcal{M}] \| \Theta[\mathcal{N}]) \leq \overline{\mathbf{C}}(\mathcal{M} \| \mathcal{N}) .
\end{aligned}
$$

3. Optimality. Any quantum channel divergence $\mathbb{D}$ that reduces to $\mathbf{C}$ on pairs of channels in $\mathfrak{R}(A \rightarrow B)$ must satisfy, for all $\mathcal{M}, \mathcal{N} \in C P T P(A \rightarrow B)$,

$$
\underline{\mathbf{C}}(\mathcal{M} \| \mathcal{N}) \leq \mathbb{D}(\mathcal{M} \| \mathcal{N}) \leq \overline{\mathbf{C}}(\mathcal{M} \| \mathcal{N}) .
$$

4. Subadditivity and Superadditivity. Suppose that $\mathbf{C}$ is weakly additive, that is, for any $k \in \mathbb{N}$

$$
\mathbf{C}\left(\mathcal{M}^{\otimes k} \| \mathcal{N}^{\otimes k}\right)=k \mathbf{C}(\mathcal{M} \| \mathcal{N}) .
$$

Then, $\underline{\mathbf{C}}$ is superadditive and $\overline{\mathbf{C}}$ is subadditive. Explicitly, for any $\mathcal{M}_{1}, \mathcal{M}_{2} \in \operatorname{CPTP}(A \rightarrow B)$ and any $\mathcal{N}_{1}, \mathcal{N}_{2} \in$ $\operatorname{CPTP}\left(A^{\prime} \rightarrow B^{\prime}\right)$,

$\underline{\mathbf{C}}\left(\mathcal{M}_{1} \otimes \mathcal{M}_{2} \| \mathcal{N}_{1} \otimes \mathcal{N}_{2}\right) \geq \underline{\mathbf{C}}\left(\mathcal{M}_{1} \| \mathcal{N}_{1}\right)+\underline{\mathbf{C}}\left(\mathcal{M}_{2} \| \mathcal{N}_{2}\right)$, $\overline{\mathbf{C}}\left(\mathcal{M}_{1} \otimes \mathcal{M}_{2} \| \mathcal{N}_{1} \otimes \mathcal{N}_{2}\right) \leq \overline{\mathbf{C}}\left(\mathcal{M}_{1} \| \mathcal{N}_{1}\right)+\overline{\mathbf{C}}\left(\mathcal{M}_{2} \| \mathcal{N}_{2}\right)$.

5. Regularization. If $\mathbf{C}$ is weakly additive under tensor products, then any weakly additive quantum channel divergence $\mathbb{D}$ that reduces to $\mathbf{C}$ on pairs of channels in $\mathfrak{R}(A \rightarrow B)$, must satisfy, for all $\mathcal{M}, \mathcal{N} \in C P T P(A \rightarrow B)$,

$$
\underline{\mathbf{C}}^{\text {reg }}(\mathcal{M} \| \mathcal{N}) \leq \mathbb{D}(\mathcal{M} \| \mathcal{N}) \leq \overline{\mathbf{C}}^{\text {reg }}(\mathcal{M} \| \mathcal{N}),
$$

where

$$
\begin{aligned}
& \underline{\mathbf{C}}^{r e g}(\mathcal{M} \| \mathcal{N})=\lim _{n \rightarrow \infty} \frac{1}{n} \underline{\mathbf{C}}\left(\mathcal{M}^{\otimes n} \| \mathcal{N}^{\otimes n}\right), \\
& \overline{\mathbf{C}}^{r e g}(\mathcal{M} \| \mathcal{N})=\lim _{n \rightarrow \infty} \frac{1}{n} \overline{\mathbf{C}}\left(\mathcal{M}^{\otimes n} \| \mathcal{N}^{\otimes n}\right),
\end{aligned}
$$

and $\underline{\mathbf{C}}^{\text {reg }}$ and $\overline{\mathbf{C}}^{\text {reg }}$ are themselves weakly additive channel divergences.
Remark.-In the case in which $\mathbf{C}$ is additive (even weakly additive), the minimal and maximal extensions are superadditive and subadditive, respectively. This, in turn, implies that the limits in Eq. (56) exist so that $\overline{\mathbf{C}}^{\text {reg }}$ and $\underline{\mathbf{C}}^{\text {reg }}$ are well defined. Moreover, in general, the bounds on $\mathbb{D}$ in Eq. (55) are tighter than the bounds in Eq. (52). This assertion follows from the fact that $\mathbf{C}$ is superadditive and, in particular, satisfies $\underline{\mathbf{C}}^{\text {reg }}(\mathcal{N} \| \mathcal{M}) \geq \underline{\mathbf{C}}(\mathcal{N} \| \mathcal{M})$. Similarly, the subadditivity of $\overline{\mathbf{C}}$ implies that $\overline{\mathbf{C}}^{\text {reg }}(\mathcal{N} \| \mathcal{M}) \leq$ $\overline{\mathbf{C}}(\mathcal{N} \| \mathcal{M})$.

In the following subsections, we apply Theorem 5 to the cases in which $\mathfrak{R}$ is the subset of all quantum states (i.e., replacement channels) and the subset of all classical states. We see that this gives rise to several optimal channel extensions of state divergences. We start, however, by using the above theorem to prove the uniqueness of the max channel relative entropy.

\section{B. Uniqueness of the max relative entropy}

The max relative entropy is defined for any $\rho, \sigma \in \mathfrak{D}(A)$ as

$$
D_{\max }(\rho \| \sigma):=\log \min \{t: t \sigma \geq \rho t \in \mathbb{R}\} .
$$

The max relative entropy is unique with respect to its monotonicity property. Unlike the relative entropy and all the other Rényi entropies, it behaves monotonically under any $\mathrm{CP}$ map (not necessarily trace preserving or trace nonincreasing). More precisely, let $\rho, \sigma \in \mathfrak{D}(A)$ and let $\mathcal{E} \in \mathrm{CP}(A \rightarrow B)$ be such that $\mathcal{E}(\rho)$ and $\mathcal{E}(\sigma)$ are normalized quantum states in $\mathfrak{D}(B)$. Since we do not assume here that $\mathcal{E}$ is trace nonincreasing, we cannot conclude that there exists a CPTP map that achieves the same task; i.e., taking the pair $(\rho, \sigma)$ to the pair $[\mathcal{E}(\rho), \mathcal{E}(\sigma)]$. Yet, the max divergence behaves monotonically under such maps; explicitly, for a given $\rho, \sigma \in \mathfrak{D}(A)$,

$$
D_{\max }(\mathcal{E}(\rho) \| \mathcal{E}(\sigma)) \leq D_{\max }(\rho \| \sigma),
$$

for any $\mathcal{E} \in \operatorname{CP}(A \rightarrow B)$ for which $\mathcal{E}(\rho), \mathcal{E}(\sigma) \in \mathfrak{D}(B)$ for the given $\rho$ and $\sigma$. Note that we assume here that $D_{\max }$ is defined only on pairs of normalized states. Extensions to subnormalized states can be made using the techniques studied in Ref. [5].

For quantum channels, $D_{\max }$ has been defined analogously to the states case, as [22]

$$
D_{\max }(\mathcal{N} \| \mathcal{M}):=\log \min \{t \in \mathbb{R}: t \mathcal{M} \geq \mathcal{N}\}
$$

One can easily see that similar to the states case, $D_{\max }(\mathcal{N} \| \mathcal{M})$ behaves monotonically under any CPpreserving (CPP) supermap that takes the pair of channels $(\mathcal{M}, \mathcal{N})$ to any other pair of channels $\left(\mathcal{M}^{\prime}, \mathcal{N}^{\prime}\right)$. Specifically, let $\Theta \in \operatorname{CPP}\left(A B \rightarrow A^{\prime} B^{\prime}\right)$ be a CPP supermap 
that is not necessarily a superchannel and suppose that $\mathcal{M}^{\prime}:=\Theta[\mathcal{N}]$ and $\mathcal{N}^{\prime}:=\Theta[\mathcal{N}]$ are quantum channels in $\operatorname{CPTP}\left(A^{\prime} \rightarrow B^{\prime}\right)$. Then,

$$
D_{\max }\left(\mathcal{M}^{\prime} \| \mathcal{N}^{\prime}\right) \leq D_{\max }(\mathcal{M} \| \mathcal{N}) .
$$

We show here that the extension of $D_{\max }$ from classical states to quantum channels is unique. We already know from Property 6 of Theorem 1 that any channel relative entropy cannot exceed $D_{\max }$. In fact, in the proof of Property 6 of Theorem 1, we only use the normalization property of relative entropy. Therefore, one can conclude something slightly stronger - that it is even not possible to extend $D_{\max }$ to a nonadditive channel divergence.

From the optimality property of Theorem 5 , it follows that in order to prove uniqueness, it is sufficient to show that the maximal and minimal extensions of $D_{\max }$ are equal to each other. Applying the general framework for extensions developed in the previous subsection, the maximal and minimal extensions of $D_{\max }$ to quantum channels, denoted by $\bar{D}_{\max }$ and $\underline{\mathrm{D}}_{\max }$, respectively, are defined for any $\mathcal{N}, \mathcal{M} \in \operatorname{CPTP}(A \rightarrow B)$ as

$$
\begin{aligned}
& \underline{\mathrm{D}}_{\max }(\mathcal{N} \| \mathcal{M}):=\sup _{\substack{|X| \in \mathbb{N} \\
\Theta \in \mathbb{S}(A B \rightarrow X)}} D_{\max }(\Theta[\mathcal{N}] \| \Theta[\mathcal{M}]) \\
& \bar{D}_{\max }(\mathcal{N} \| \mathcal{M}):=\inf _{\substack{|X| \in \mathbb{N}, \mathbf{p}, \mathbf{q} \in \mathcal{D}(X) \\
\mathcal{N}=\Theta[\mathbf{p}], \mathcal{M}=\Theta[\mathbf{q}] \\
\Theta \in \mathfrak{S}(X \rightarrow A B)}} D_{\max }(\mathbf{p} \| \mathbf{q}) .
\end{aligned}
$$

Theorem 6 (Uniqueness of the Dynamical Max Relative Entropy). Let $\mathbb{D}$ be a channel divergence that reduces to $D_{\max }$ on classical probability distributions; i.e., for any classical system $X$ and $\mathbf{p}, \mathbf{q} \in \mathfrak{D}(X), \mathbb{D}(\mathbf{p} \| \mathbf{q})=$ $D_{\max }(\mathbf{p} \| \mathbf{q})$. Then, for all $\mathcal{N}, \mathcal{M} \in C P T P(A \rightarrow B)$,

$$
\mathbb{D}(\mathcal{N} \| \mathcal{M})=D_{\max }(\mathcal{N} \| \mathcal{M})
$$

Remark.-Note that we do not assume that $\mathbb{D}$ is a channel relative entropy (i.e., additive) but only a divergence that reduces to the max relative entropy on classical states. The main idea of the proof is to show that the two expressions in Eq. (61) are both equal to $D_{\max }$.

\section{Extension from a quantum (state) divergence to a channel divergence}

In this section, we study the optimal extensions of quantum state divergences to quantum channel divergences. For a given quantum state divergence $\mathbf{D}$, we denote by $\underline{\mathbf{D}}$ its minimal channel extension. According to Eq. (48), the minimal channel extension is given by

$$
\begin{gathered}
\underline{\mathbf{D}}(\mathcal{N} \| \mathcal{M}):=\sup _{\Theta \in \mathfrak{S}\left(A B \rightarrow R^{\prime}\right)} \mathbf{D}(\Theta[\mathcal{N}] \| \Theta[\mathcal{M}])=\sup _{\substack{\mathcal{E} \in \operatorname{CPTP} \\
\psi \in \mathfrak{D}(R A)}} \\
\mathbf{D}\left[\mathcal{E}^{B R \rightarrow R^{\prime}} \circ \mathcal{N}^{A \rightarrow B}\left(\psi^{A R}\right) \| \mathcal{E}^{B R \rightarrow R^{\prime}} \circ \mathcal{M}^{A \rightarrow B}\left(\psi^{A R}\right)\right] \\
\quad=\max _{\psi \in \mathfrak{D}(R A)} \mathbf{D}\left[\mathcal{N}^{A \rightarrow B}\left(\psi^{A R}\right) \| \mathcal{M}^{A \rightarrow B}\left(\psi^{A R}\right)\right]
\end{gathered}
$$

where in the last equality the supremum has been replaced with a maximum, since without loss of generality we can assume that $|R|=|A|$ and that $\psi^{R A}$ is a pure state [14,15].

Similarly, by the definition in Eq. (49), the maximal channel extension is given by

$$
\begin{gathered}
\overline{\mathbf{D}}(\mathcal{N} \| \mathcal{M}) \\
:=\inf _{\substack{\Theta \in \mathfrak{S}(R \rightarrow A B) \\
\rho, \sigma \in \mathcal{D}(R)}}\{\mathbf{D}(\rho \| \sigma): \mathcal{N}=\Theta[\rho], \mathcal{M}=\Theta[\sigma]\} \\
=\inf _{\substack{\mathcal{E} \in \operatorname{CPTP}(R A \rightarrow B) \\
\rho, \sigma \in \mathfrak{D}(R)}}\left\{\mathbf{D}(\rho \| \sigma): \mathcal{N}=\mathcal{E}_{\rho}, \mathcal{M}=\mathcal{E}_{\sigma}\right\},
\end{gathered}
$$

where for any density matrices $\rho, \sigma \in \mathfrak{D}(R)$ and channel $\mathcal{E} \in \operatorname{CPTP}(R A \rightarrow B)$, we denote

$$
\begin{aligned}
& \mathcal{E}_{\rho}^{A \rightarrow B}\left(\omega^{A}\right)=\mathcal{E}^{R A \rightarrow B}\left(\rho^{R} \otimes \omega^{A}\right), \\
& \mathcal{E}_{\sigma}^{A \rightarrow B}\left(\omega^{A}\right)=\mathcal{E}^{R A \rightarrow B}\left(\sigma^{R} \otimes \omega^{A}\right) \quad \forall \omega \in \mathfrak{L}(A) .
\end{aligned}
$$

The above channels have been studied under the name environment-parametrized channels [45-47], and the expression in Eq. (63) has been used in the literature for the cases in which $\mathbf{D}$ is the trace norm (in which case, D becomes the diamond norm [48]), Umegaki relative entropy, and quantum Rényi divergences [14,15]. The following corollary is the restatement of Theorem 5 for the optimal channel extensions of quantum state divergences.

Corollary 1. Let $\mathbf{D}$ be a quantum (state) divergence and let $\underline{\mathbf{D}}$ and $\overline{\mathbf{D}}$ be its minimal and maximal extensions to quantum channels. Then,

1. Both $\underline{\mathbf{D}}$ and $\overline{\mathbf{D}}$ are quantum-channel divergences.

2. Both $\underline{\mathbf{D}}$ and $\overline{\mathbf{D}}$ reduce to $\mathbf{D}$ on quantum states.

3. Any other channel divergences $\mathbb{D}$ that reduce to $\mathbf{D}$ on quantum states must satisfy, for all $\mathcal{N}, \mathcal{M} \in C P T P$ $(A \rightarrow B)$,

$$
\underline{\mathbf{D}}(\mathcal{N} \| \mathcal{M}) \leq \mathbb{D}(\mathcal{N} \| \mathcal{M}) \leq \overline{\mathbf{D}}(\mathcal{N} \| \mathcal{M}) .
$$

4. If $\mathbf{D}$ is a weakly additive quantum state divergence, then $\mathbf{D}$ is superadditive and $\overline{\mathbf{D}}$ is subadditive with 
respect to tensor products. Explicitly, for any $\mathcal{M}_{1}, \mathcal{M}_{2} \in$ $C P T P(A \rightarrow B)$ and any $\mathcal{N}_{1}, \mathcal{N}_{2} \in \operatorname{CPTP}\left(A^{\prime} \rightarrow B^{\prime}\right)$,

$$
\begin{aligned}
& \underline{\mathbf{D}}\left(\mathcal{M}_{1} \otimes \mathcal{M}_{2} \| \mathcal{N}_{1} \otimes \mathcal{N}_{2}\right) \geq \underline{\mathbf{D}}\left(\mathcal{M}_{1} \| \mathcal{N}_{1}\right)+\underline{\mathbf{D}}\left(\mathcal{M}_{2} \| \mathcal{N}_{2}\right), \\
& \overline{\mathbf{D}}\left(\mathcal{M}_{1} \otimes \mathcal{M}_{2} \| \mathcal{N}_{1} \otimes \mathcal{N}_{2}\right) \leq \overline{\mathbf{D}}\left(\mathcal{M}_{1} \| \mathcal{N}_{1}\right)+\overline{\mathbf{D}}\left(\mathcal{M}_{2} \| \mathcal{N}_{2}\right) .
\end{aligned}
$$

5. If $\mathbf{D}$ is a weakly additive quantum state divergence, then any weakly additive quantum channel divergence $\mathbb{D}$ that reduces to $\mathbf{D}$ on quantum states must satisfy, for all $\mathcal{M}, \mathcal{N} \in \operatorname{CPTP}(A \rightarrow B)$,

$$
\underline{\mathbf{D}}^{\text {reg }}(\mathcal{M} \| \mathcal{N}) \leq \mathbb{D}(\mathcal{M} \| \mathcal{N}) \leq \overline{\mathbf{D}}^{\text {reg }}(\mathcal{M} \| \mathcal{N}),
$$

where

$$
\begin{aligned}
& \underline{\mathbf{D}}^{r e g}(\mathcal{M} \| \mathcal{N})=\lim _{n \rightarrow \infty} \frac{1}{n} \underline{\mathbf{D}}\left(\mathcal{M}^{\otimes n} \| \mathcal{N}^{\otimes n}\right), \\
& \overline{\mathbf{D}}^{\text {reg }}(\mathcal{M} \| \mathcal{N})=\lim _{n \rightarrow \infty} \frac{1}{n} \overline{\mathbf{D}}\left(\mathcal{M}^{\otimes n} \| \mathcal{N}^{\otimes n}\right)
\end{aligned}
$$

and $\underline{\mathbf{D}}^{\text {reg }}$ and $\overline{\mathbf{D}}^{\text {reg }}$ are themselves weakly additive normalized channel divergences.

In addition to the above corollary, we have the following property for the maximal extension.

Theorem 7. Let $\mathbf{D}$ be a jointly convex quantum divergence. Then, its maximal channel extension $\overline{\mathbf{D}}$ is also jointly convex.

The channel divergence $\underline{\mathbf{D}}$ has been shown in Ref. [21] to satisfy the generalized DPI. For the case in which $\mathbf{D}=$ $D$ is the Umegaki relative entropy, it has been shown in Ref. [29] that it satisfies a chain rule. The latter property in particular implies that its regularization can be expressed as [29]

$$
\begin{aligned}
& \underline{\mathrm{D}}^{\mathrm{reg}}(\mathcal{N} \| \mathcal{M})=\sup _{\rho, \sigma \in \mathfrak{D}(R A)} \\
& \left\{D\left[\mathcal{M}^{A \rightarrow B}\left(\rho^{R A}\right) \| \mathcal{N}^{A \rightarrow B}\left(\sigma^{R A}\right)\right]-D\left(\rho^{R A} \| \sigma^{R A}\right)\right\},
\end{aligned}
$$

where the expression on the right-hand side is known as the amortized divergence [16]. We see below that any channel relative entropy that reduces to the Kullback-Leibler divergence on classical states must be no smaller than the above expression.

\section{Extensions from classical state divergences to channel divergences}

In this subsection, we study optimal channel extensions of a classical state divergence $\mathscr{D}$. We define the following four optimal extensions of $\mathscr{D}$ to quantum channel divergences.
Definition 3: Let $\mathscr{D}: \mathfrak{D}(X) \times \mathfrak{D}(X) \rightarrow \mathbb{R}_{+}$be a classical (state) divergence and let $\mathcal{N}, \mathcal{M} \in \operatorname{CPTP}(A \rightarrow B)$ be two quantum channels. We define four extensions of $\mathscr{D}$ to quantum channels :

1. The minimal extension of $\mathscr{D}$,

$$
\underline{\mathscr{D}}(\mathcal{N} \| \mathcal{M}):=\sup _{\substack{|X| \in \mathbb{N} \\ \Theta \in \mathfrak{S}(A B \rightarrow X)}} \mathscr{D}(\Theta[\mathcal{N}] \| \Theta[\mathcal{M}])
$$

2. The maximal extension of $\mathscr{D}$,

$$
\overline{\mathscr{D}}(\mathcal{N} \| \mathcal{M}):=\inf _{\substack{|X| \in \mathbb{N}, \mathbf{p}, \mathbf{q} \in \mathfrak{D}(X) \\ \mathcal{N}=\Theta[\mathbf{p}], \mathcal{M}=\Theta[\mathbf{q}] \\ \Theta \in \mathfrak{S}(X \rightarrow A B)}} \mathscr{D}(\mathbf{p} \| \mathbf{q})
$$

3. The geometric extension of $\mathscr{D}$,

$$
\widehat{\mathscr{D}}(\mathcal{N} \| \mathcal{M}):=\sup _{\substack{|R| \in \mathbb{N} \\ \Theta \in \mathfrak{S}(A B \rightarrow R)}} \overline{\mathscr{D}}(\Theta[\mathcal{N}] \| \Theta[\mathcal{M}])
$$

where $\overline{\mathscr{D}}$ is the maximal quantum state extension of $\mathscr{D}$.

4. The min-max extension of $\mathscr{D}$,

$$
\underline{\mathscr{D}}^{\uparrow}(\mathcal{N} \| \mathcal{M}):=\inf _{\substack{|R| \in \mathbb{N}, \rho, \sigma \in \mathfrak{Q}(R) \\ \mathcal{N}=\Theta[\rho], \mathcal{M}=\Theta[\sigma] \\ \Theta \in \mathfrak{S}(R \rightarrow A B)}} \mathscr{D}(\rho \| \sigma),
$$

where $\underline{\mathscr{D}}$ is the minimal quantum state extension of $\mathscr{D}$.

From Theorem 5, it follows that all the above four functions satisfy the generalized data processing inequality (and therefore they are indeed divergences) and they all reduce to the classical divergence $\mathscr{D}$ on classical states. Moreover, for a pair of quantum states $\rho, \sigma \in \mathfrak{D}(A)$, we have $\widehat{\mathscr{D}}(\rho \| \sigma)=\overline{\mathscr{D}}(\rho \| \sigma)$ and $\underline{\mathscr{D}}^{\uparrow}(\rho \| \sigma)=\underline{\mathscr{D}}(\rho \| \sigma)$. In addition, Theorem 5 implies that any channel divergence $\mathbb{D}$ that reduces on classical states to a classical divergence $\mathscr{D}$ must satisfy, for all $\mathcal{N}, \mathcal{M} \in \operatorname{CPTP}(A \rightarrow B)$,

$$
\underline{\mathscr{D}}(\mathcal{N} \| \mathcal{M}) \leq \mathbb{D}(\mathcal{N} \| \mathcal{M}) \leq \overline{\mathscr{D}}(\mathcal{N} \| \mathcal{M})
$$

This applies, in particular, to the cases $\mathbb{D}=\widehat{\mathscr{D}}$ and $\mathbb{D}=$ $\underline{D}^{\uparrow}$ and we also note that, in general, the optimal channel extensions in Eqs. (63) and (65) of a quantum state divergence $\mathbf{D}$, which reduces to a classical state divergence $\mathscr{D}$ on classical states, satisfy, for all $\mathcal{M}, \mathcal{N} \in \operatorname{CPTP}(A \rightarrow B)$,

$$
\underline{\mathscr{D}}(\mathcal{M} \| \mathcal{N}) \leq \underline{\mathbf{D}}(\mathcal{M} \| \mathcal{N}) \leq \overline{\mathbf{D}}(\mathcal{M} \| \mathcal{N}) \leq \overline{\mathscr{D}}(\mathcal{M} \| \mathcal{N}) .
$$




\section{The minimal channel extension and its regularization}

Note that the minimal extension $\mathscr{D}$ can be expressed as

$$
\begin{aligned}
& \underline{\mathscr{D}}(\mathcal{N} \| \mathcal{M}):=\sup \mathscr{D}\left[\mathcal{E}_{B R \rightarrow X} \circ \mathcal{N}_{A \rightarrow B}\left(\psi_{A R}\right) \|\right. \\
& \left.\quad \times \mathcal{E}_{B R \rightarrow X} \circ \mathcal{M}_{A \rightarrow B}\left(\psi_{A R}\right)\right]
\end{aligned}
$$

where the supremum is over all systems $X, R$, over all $\psi \in \mathfrak{D}(A R)$, and over all $\mathcal{E} \in \operatorname{CPTP}(B R \rightarrow X)$. Note that, without loss of generality, we can assume that $\psi_{R A}$ is a pure state. There is at least one divergence for which the above expression coincides with the optimization given in Eq. (63).

Theorem 8. Let $\mathcal{M}, \mathcal{N} \in \operatorname{CPTP}(A \rightarrow B)$ and for any $\epsilon \in$ $[0,1)$, let $\mathscr{D}=D_{\min }^{\epsilon}$ be the classical hypothesis-testing divergence (i.e., the classical version of the one defined in Eq. (10)). Then,

$$
\begin{aligned}
& \underline{\mathscr{D}}(\mathcal{M} \| \mathcal{N})=D_{\min }^{\epsilon}(\mathcal{M} \| \mathcal{N}) \\
& \quad:=\sup _{\psi \in \mathfrak{D}(R A)} D_{\min }^{\epsilon}\left[\mathcal{M}^{A \rightarrow B}\left(\psi^{R A}\right) \| \mathcal{N}^{A \rightarrow B}\left(\psi^{R A}\right)\right] .
\end{aligned}
$$

Remark.-The above theorem implies that any channel divergence $\mathbb{D}$ that reduces to $D_{\min }^{\epsilon}$ on classical states satisfies, for all $\mathcal{M}, \mathcal{N} \in \operatorname{CPTP}(A \rightarrow B)$,

$$
\mathbb{D}(\mathcal{M} \| \mathcal{N}) \geq D_{\text {min }}^{\epsilon}(\mathcal{M} \| \mathcal{N}) .
$$

The minimal channel extension is typically not additive even if the classical divergence $\mathscr{D}$ is additive (i.e., $\mathscr{D}$ is a relative entropy). However, from Theorem 5, we know that if $\mathscr{D}$ is a classical relative entropy, then its channel extension $\mathscr{D}$ is superadditive. This means that the limit in its regularization exists;

$$
\underline{\mathscr{D}}^{\mathrm{reg}}(\mathcal{N} \| \mathcal{M}):=\lim _{n \rightarrow \infty} \frac{1}{n} \underline{\mathscr{D}}\left(\mathcal{N}^{\otimes n} \| \mathcal{M}^{\otimes n}\right) .
$$

From Theorem 5, it follows that $\underline{\mathscr{D}}^{\text {reg }}$ is a weakly additive divergence and all channel relative entropies that reduce to $\mathscr{D}$ on classical states must be no smaller than $\mathscr{D}^{\text {reg }}$.

Now suppose that $\mathscr{D}=D$ is the KL divergence. In this case, we denote by $\underline{D}^{\text {reg }}$ its minimal regularized channel extension. Another closely related quantity that plays an important role in applications is the regularized version of the minimal channel extension of the (quantum) Umegaki relative entropy, denoted as $D^{\text {reg }}$. That is,

$$
D^{\mathrm{reg}}(\mathcal{M} \| \mathcal{N}):=\lim _{n \rightarrow \infty} \frac{1}{n} D\left(\mathcal{M}^{\otimes n} \| \mathcal{N}^{\otimes n}\right),
$$

where

$$
D(\mathcal{M} \| \mathcal{N}):=\sup _{\psi \in \mathfrak{D}(R A)} D\left[\mathcal{M}^{A \rightarrow B}\left(\psi^{A R}\right) \| \mathcal{N}^{A \rightarrow B}\left(\psi^{A R}\right)\right]
$$

in which $D$ is the Umegaki relative entropy. Since $\underline{D}^{\text {reg }}$ is the minimal channel extension, we must have

$$
\underline{\mathrm{D}}^{\mathrm{reg}}(\mathcal{M} \| \mathcal{N}) \leq D^{\mathrm{reg}}(\mathcal{M} \| \mathcal{N}) .
$$

Note that if $\mathcal{M}$ and $\mathcal{N}$ in the above equation are quantum states (i.e., the input dimension $|A|=1$ ), then the equality holds. This is due to the fact that on quantum states, the Umegaki relative entropy $D^{\text {reg }}(\rho \| \sigma)=D(\rho \| \sigma)$ equals the minimal additive state extension of the KL relative entropy [see Eq. (12)]. We now show that the equality also holds for any two channels.

Theorem 9. Let $\mathcal{M}, \mathcal{N} \in \operatorname{CPTP}(A \rightarrow B)$ be two quantum channels. Then,

$$
\underline{D}^{r e g}(\mathcal{M} \| \mathcal{N})=D^{r e g}(\mathcal{M} \| \mathcal{N}) \text {. }
$$

That is, $D^{\text {reg }}(\mathcal{M} \| \mathcal{N})$ is the smallest channel relative entropy that reduces to the KL relative entropy on classical states.

Starting with a classical relative entropy $\mathscr{D}$, the process at which we arrived at the weakly additive channel divergence $\mathscr{D}^{\text {reg }}$ had two steps: (1) extend $\mathscr{D}$ to the minimal channel divergence $\mathscr{D}$ and (2) regularize $\mathscr{D}$ to obtain a weakly additive divergence. One can also introduce regularization in the state level and only then apply the channel extension. Specifically, starting with a classical relative entropy $\mathscr{D}$, we apply the following four steps:

1. Extend $\mathscr{D}$ to the minimal quantum state divergence $\underline{\mathscr{D}}$.

2. Regularize $\mathscr{D}$ to obtain a weakly additive quantum state divergence $\underline{\mathscr{D}}^{\text {reg }}$.

3. Using the minimal extension, extend $\underline{\mathscr{D}}^{\text {reg }}$ to a channel divergence $\mathscr{D}_{\mathrm{ch}}$.

4. Regularize $\mathscr{\mathscr { D }}_{\text {ch }}$ to obtain a weakly additive channel divergence $\underline{\mathscr{D}}_{\mathrm{ch}}^{\text {reg }}$.

Figure 3 illustrate these four steps.

In Ref. [10], it has been shown that if $\mathscr{D}$ is the classical Rényi entropy with $\alpha \in[1 / 2, \infty]$, then $\mathscr{D}^{\text {reg }}$ is the sandwiched quantum relative entropy of order $\alpha[8,9]$. Therefore, in this case, $\mathscr{D}_{\mathrm{ch}}(\mathcal{M} \| \mathcal{N})=D_{\alpha}(\mathcal{M} \| \mathcal{N})$ is simply the channel extension of the sandwich relative entropy so that $\mathscr{D}_{\text {ch }}^{\text {reg }}(\mathcal{M} \| \mathcal{N})=D_{\alpha}^{\text {reg }}(\mathcal{M} \| \mathcal{N})$ is just the regularization of $D_{\alpha}(\mathcal{M} \| \mathcal{N})$. In Theorem 9 above, we show that for $\alpha=1, D_{\alpha}^{\text {reg }}(\mathcal{M} \| \mathcal{N})=\underline{D}_{\alpha}^{\text {reg }}(\mathcal{M} \| \mathcal{N})$, which means that for $\alpha=1, D_{\alpha}^{\text {reg }}$ is the smallest weakly additive divergence that reduces to the KL relative entropy on classical states. The question of whether this equality holds for all $\alpha \in[1 / 2, \infty]$ remains open. 


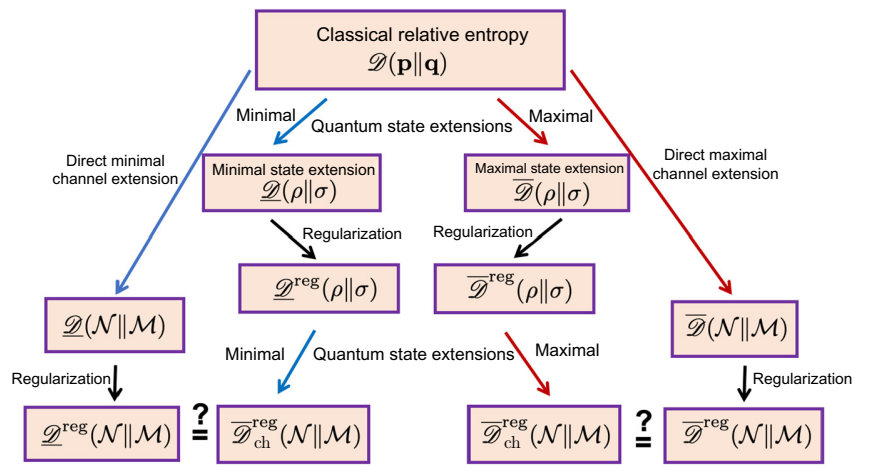

FIG. 3. The order between extensions and regularizations matters.

\section{The maximal channel extension}

The maximal channel extension in Eq. (73) is new and has not been studied before. Note that the infimum in Eq. (73) can be expressed as

$$
\overline{\mathscr{D}}(\mathcal{M} \| \mathcal{N}):=\inf _{\substack{|X| \in \mathbb{N}, \mathbf{p}, \mathbf{q} \in \mathcal{D}(X) \\ \mathcal{M}=\sum_{x}, p_{\mathcal{E}}, \mathcal{\mathcal { E }}=\sum_{x} q_{x} \mathcal{E}_{x} \\\left\{\mathcal{E}_{x}\right\} \subset \operatorname{CPTP}(A \rightarrow B)}} \mathscr{D}(\mathbf{p} \| \mathbf{q}) .
$$

For the case in which $\mathcal{M}$ is an isometry, we obtain the following result.

Theorem 10. Let $\mathcal{V} \in \operatorname{CPTP}(A \rightarrow B)$ be an isometry channel defined via $\mathcal{V}(\rho)=V \rho V^{*}$, for all $\rho \in \mathfrak{D}(A)$, and with isometry matrix $V$ (i.e., $V^{*} V=I^{A}$ ). Then, for any $\mathcal{N} \in \operatorname{CPTP}(A \rightarrow B)$,

$$
\overline{\mathscr{D}}(\mathcal{V} \| \mathcal{N})=D_{\max }(\mathcal{V} \| \mathcal{N})=\log \operatorname{Tr}\left[J_{\mathcal{N}}^{-1} J_{\mathcal{V}}\right]
$$

In Sec. III B, we provide a closed formula of this divergence for the classical case. We see that the formula reveals that this divergence is not additive (even for classical channels) and therefore is not a relative entropy. Recall, however, that from Theorem 5, we know that if $\mathscr{D}$ is a classical relative entropy, then its channel extension $\overline{\mathscr{D}}$ is subadditive. This means that the limit in its regularization exists and $\overline{\mathscr{D}}^{\text {reg }} \leq \overline{\mathscr{D}}$. The divergence $\overline{\mathscr{D}}^{\text {reg }}$ is weakly additive and it remains open to determine whether it is a relative entropy (i.e., fully additive).

\section{The geometric channel relative entropy}

Given a classical divergence $\mathscr{D}$, its maximal extension to quantum states is given for all $\rho, \sigma \in \mathfrak{D}(A)$ by

$$
\overline{\mathscr{D}}(\rho \| \sigma):=\inf _{\substack{|X| \in \mathbb{N}, \mathbf{p}, \mathbf{q} \in \mathcal{D}(X) \\ \mathcal{E}(\mathbf{p})=\rho, \mathcal{E}(\mathbf{q})=\sigma \\ \mathcal{E} \in \operatorname{CPTP}(X \rightarrow A)}} \mathscr{D}(\mathbf{p} \| \mathbf{q}) .
$$

The geometric divergence is defined as the minimal channel extension of this maximal state extension of $\mathscr{D}$. It can be expressed as [cf. Eq. (74)]

$$
\widehat{\mathscr{D}}(\mathcal{M} \| \mathcal{N}):=\sup _{\psi \in \mathfrak{D}(R A)} \overline{\mathscr{D}}\left[\mathcal{M}^{A \rightarrow B}\left(\psi^{R A}\right) \| \mathcal{N}^{A \rightarrow B}\left(\psi^{R A}\right)\right]
$$

For the case in which $\mathscr{D}=D_{\alpha}$ is the classical Rényi entropy with $\alpha \in(0,2]$ and $\alpha \neq 1$, it has been proved in Refs. $[18,19]$ that

$$
\widehat{\mathscr{D}}(\mathcal{M} \| \mathcal{N})=\widehat{D}_{\alpha}(\mathcal{M} \| \mathcal{N}):=\frac{1}{\alpha-1} \log \widehat{Q}_{\alpha}(\mathcal{M} \| \mathcal{N}),
$$

where

with $J_{\mathcal{M}_{\epsilon}}^{A B}:=J_{\mathcal{M}}^{A B}+\epsilon I^{A B}$ and

$$
G_{\alpha}(X, Y):=Y^{1 / 2}\left(Y^{-\frac{1}{2}} X Y^{-\frac{1}{2}}\right)^{\alpha} Y^{1 / 2} \quad \forall X, Y>0 .
$$

For $\alpha=1$, it is given by

$$
\begin{aligned}
\widehat{D}_{\alpha=1}(\mathcal{M} \| \mathcal{N})=\widehat{D}(\mathcal{M} \| \mathcal{N}) \\
\quad:= \begin{cases}\left\|\operatorname{Tr}_{B}\left[\widehat{G}\left(J_{\mathcal{M}}^{A B}, J_{\mathcal{N}}^{A B}\right)\right]\right\|_{\infty}, & \text { if } \operatorname{supp}\left(J_{\mathcal{M}}^{A B}\right) \subseteq \operatorname{supp}\left(J_{\mathcal{N}}^{A B}\right), \\
\infty, & \text { otherwise }\end{cases}
\end{aligned}
$$


where

$$
\widehat{G}(X, Y):=X^{1 / 2} \log \left(X^{1 / 2} Y^{-1} X^{1 / 2}\right) X^{1 / 2} .
$$

For all $\alpha \in(0,2]$, the above formula gives, for an isometry $\mathcal{V}$ and a channel $\mathcal{N}$

$$
\widehat{D}_{\alpha}(\mathcal{V} \| \mathcal{N})=D_{\max }(\mathcal{V} \| \mathcal{N}) .
$$

Hence, due to Theorem 10, $\widehat{D}_{\alpha}(\mathcal{V} \| \mathcal{N})$ coincides with the maximal quantum-channel extension $\bar{D}_{\alpha}(\mathcal{V} \| \mathcal{N})$. However, recall that in general we have $\widehat{D}_{\alpha}(\mathcal{M} \| \mathcal{N}) \leq$ $\bar{D}_{\alpha}(\mathcal{M} \| \mathcal{N})$ and that we see in Sec. III B that the inequality can be strict even on classical channels. In fact, since $\widehat{D}_{\alpha}$ is additive, we must have $\widehat{D}_{\alpha}(\mathcal{M} \| \mathcal{N}) \leq \bar{D}_{\alpha}^{\text {reg }}(\mathcal{M} \| \mathcal{N})$, and it is left open whether this inequality can be strict for some choices of $\mathcal{M}$ and $\mathcal{N}$.

The formula (90) reveals that $\widehat{D}_{\alpha}$ is additive (at least for $\alpha \in(0,2])$. In fact, with the exception of $D_{\max }$, this function is the only known channel relative entropy, since all other channel divergences discussed in this paper are at most known to be weakly additive and the question of whether they are fully additive is open. In Ref. [18], $\widehat{D}_{\alpha}$ has been used to derive upper bounds on certain quantum information processing tasks and in Ref. [19] it has been used to upper bound some optimal rates in the context of channel discrimination.

\section{OUTLOOK}

\section{A. Some applications of channel divergences}

One of the fundamental tasks in quantum-information theory is the distinguishability of two quantum channels $\mathcal{N}, \mathcal{M} \in \operatorname{CPTP}(A \rightarrow B)$. In Ref. [25], it has been shown that the asymptotically optimal discrimination rate of the type-II error exponent for parallel strategy is given by $D^{\mathrm{reg}}(\mathcal{N} \| \mathcal{M})$ and for the adaptive strategy by the amortized divergence, as given in Eq. (71). Remarkably, in Ref. [29] it has been shown that the amortized divergence equals $D^{\text {reg }}$, indicating that both the parallel and adaptive strategies yield the same error exponent.

Despite the physical significance of $D^{\mathrm{reg}}(\mathcal{N} \| \mathcal{M})$ for channel discrimination, we know very little about this quantity due to the fact that it is defined in terms of an optimization over a noncompact domain. Therefore, in such scenarios, it is often useful to study the properties of such uncomputable functions.

It is known [43] that $D^{\text {reg }}(\mathcal{N} \| \mathcal{M})$ is an additive function and therefore it fits our definition of a relative entropy. This immediately implies that it satisfies all the properties outlined in Theorem 1. In particular, it satisfies the continuity properties given in Eqs. (34) and (33), indicating that if two channels $\mathcal{N}, \mathcal{N}^{\prime} \in \operatorname{CPTP}(A \rightarrow B)$ are close (in terms of the Thompson metric $D_{T}$ ), then they have similar discrimination rates with any other channel
$\mathcal{M} \in \operatorname{CPTP}(A \rightarrow B)$. We point out that even though the Umegaki relative entropy is continuous (in fact, asymptotically continuous) one cannot conclude that these properties carry over to $D^{\text {reg }}$ due to the optimization involved with unbounded dimensions.

Theorem 9 establishes that $D^{\mathrm{reg}}(\mathcal{N} \| \mathcal{M})$ is the smallest channel relative entropy among all channel relative entropies that reduces to the KL divergence on classical states. This means that it is not possible to find a computable lower bound for $D^{\text {reg }}(\mathcal{N} \| \mathcal{M})$ that is itself a channel divergence. On the other hand, the geometric channel relative entropy $\widehat{D}$ provides such an upper bound [18].

Channel relative entropies have several other applications in quantum Shannon theory. For example, consider two distant parties, Alice and Bob, who share a classicalquantum channel $\mathcal{N} \in \operatorname{CPTP}(X \rightarrow B)$. This channel can be used to transmit classical information at a rate that is given by the Holevo information (see, e.g., Ref. [3]). Interestingly, the Holevo information $\chi(\mathcal{N})$ can be expressed as

$$
\chi\left(\mathcal{N}^{X \rightarrow B}\right)=\min _{\mathcal{E}} D\left(\mathcal{N}^{X \rightarrow B} \| \mathcal{E}^{X \rightarrow B}\right),
$$

where the minimum is over all replacement channels of the form $\mathcal{E}(|x\rangle\langle x|)=\sigma^{B}$ for all $x$ and a fixed $\sigma \in \mathfrak{D}(B)$. We can therefore interpret the Holevo quantity as a channel divergence distance to the set of "free" channels. In this context, the only free channels are the replacement channels. Also, the entanglement-assisted classical communication rate can be expressed in a similar fashion. These examples indicate that in the channel domain also, divergences and relative entropies as defined in this paper are expected to play a key role in the recently active developing field of dynamical quantum resource theories [14-18,18-29].

\section{B. Conclusions}

In this paper, we introduce an axiomatic approach to dynamical divergences. This approach is minimalistic in the sense that we only require channel divergences to satisfy the generalized DPI under superchannels and channel relative entropies to be, in addition, additive and normalized. Remarkably, we show that these axioms are sufficient to induce enough structure, leading to numerous properties satisfied by all channel relative entropies. One of our main results is a uniqueness theorem, Theorem 4, in which we show that in the classical domain, there exists only one channel relative entropy, which reduces to the Kullback-Leibler divergence on classical states (i.e., probability vectors). In the quantum case, it is known that this uniqueness does not hold even for quantum states but we are able to show that the amortized relative entropy as defined in Eq. (71) is the smallest channel relative entropy that reduces to the Kullback-Leibler divergence on classical states. Due to the one-to-one correspondence between 
classical entropies and classical relative entropies [4], this means that the amortized relative entropy is in fact the smallest one that reduces on a pair of classical states $(\mathbf{p}, \mathbf{u})$ (here, $\mathbf{u}$ is the uniform distribution) to the $\log$ of the dimension minus the Shannon entropy.

There are many open problems for future investigations. For example, in the classical domain, for $\alpha \neq 1$, is the classical-channel extension of the $\alpha$-Rényi relative entropy unique? Another interesting problem is whether the regularization of the maximal channel extension of relative entropies coincides with the geometric channel relative entropies. Finally, another interesting question is whether the maximal channel extensions of relative entropies satisfy a "chain rule" similar to the one satisfied by the minimal channel extension given in Eq. (63) [29].

\section{ACKNOWLEDGMENTS}

I would like to thank Marco Tomamichel for numerous fruitful discussions on the subject of this paper. I am also grateful for many fruitful discussions with Nilanjana Datta, Kun Fang, Xin Wang, and Mark Wilde on topics related to this work. I acknowledge support from the Natural Sciences and Engineering Research Council of Canada (NSERC).

[1] T. M. Cover and J. A. Thomas, Elements of Information Theory, Wiley Series in Telecommunications and Signal Processing (Wiley-Interscience, 2006).

[2] D. Pérez-García, M. M. Wolf, D. Petz, and M. B. Ruskai, Contractivity of positive and trace-preserving maps under lp norms, J. Math. Phys. 47, 083506 (2006).

[3] M. M. Wilde, Quantum Information Theory (Cambridge University Press, Cambridge, 2017), 2nd ed.

[4] G. Gour and M. Tomamichel, arXiv:2006.11164v1 (2020).

[5] G. Gour and M. Tomamichel, arXiv:2006.12408v2 (2020).

[6] H. Umegaki, Conditional expectation in an operator algebra. iv. entropy and information, Kodai Math. Sem. Rep. 14, 59 (1962).

[7] D. Petz, Quasi-entropies for finite quantum systems, Rep. Math. Phys. 23, 57 (1986).

[8] M. M. Wilde, A. Winter, and D. Yang, Strong converse for the classical capacity of entanglement-breaking and hadamard channels via a sandwiched rényi relative entropy, Commun. Math. Phys. 331, 593 (2014).

[9] M. Müller-Lennert, F. Dupuis, O. Szehr, S. Fehr, and M. Tomamichel, On quantum rényi entropies: A new generalization and some properties, J. Math. Phys. 54, 122203 (2013).

[10] M. Tomamichel, Quantum Information Processing with Finite Resources: Mathematical Foundations, Springer Briefs in Mathematical Physics (Springer International Publishing, 2015).

[11] K. Matsumoto, A New Quantum Version of f-Divergence, arXiv:1311.4722v4 (2018).
[12] F. Hiai and M. Mosonyi, Different quantum f-divergences and the reversibility of quantum operations, Rev. Math. Phys. 29, 1750023 (2017).

[13] N. Datta, Min- and max-relative entropies and a new entanglement monotone, IEEE Trans. Inf. Theory 55, 2816 (2009).

[14] T. Cooney, M. Mosonyi, and M. M. Wilde, Strong converse exponents for a quantum channel discrimination problem and quantum-feedback-assisted communication, Commun. Math. Phys. 344, 797 (2016).

[15] F. Leditzky, E. Kaur, N. Datta, and M. M. Wilde, Approaches for Approximate Additivity of the Holevo Information of Quantum Channels, Phys. Rev. A 97, 012332 (2018).

[16] M. Berta, C. Hirche, E. Kaur, and M. M. Wilde, Amortized Channel Divergence for Asymptotic Quantum Channel Discrimination, arXiv:1808.01498 (2018).

[17] G. Gour, Comparison of quantum channels by superchannels, IEEE Trans. Inf. Theory 65, 5880 (2019).

[18] K. Fang and H. Fawzi, Geometric rényi divergence and its applications in quantum channel capacities, arXiv:1909.05758 (2019).

[19] V. Katariya and M. M. Wilde, Geometric Distinguishability Measures Limit Quantum Channel Estimation and Discrimination, arXiv:2004.10708 (2020).

[20] E. Kaur and M. M. Wilde, Amortized entanglement of a quantum channel and approximately teleportationsimulable channels, J. Phys. A: Math. Theor. 51, 035303 (2018).

[21] G. Gour and M. M. Wilde, Entropy of a Quantum Channel, arXiv:1808.06980 (2018).

[22] Z.-W. Liu and A. Winter, Resource Theories of Quantum Channels and the Universal Role of Resource Erasure, arXiv:1904.04201 (2019).

[23] Y. Liu and X. Yuan, Operational resource theory of quantum channels, Phys. Rev. Res. 2, 012035 (2020).

[24] G. Gour and A. Winter, How to Quantify a Dynamical Quantum Resource, Phys. Rev. Lett. 123, 150401 (2019).

[25] X. Wang and M. M. Wilde, Resource theory of asymmetric distinguishability for quantum channels, Phys. Rev. Res. 1, 033169 (2019).

[26] X. Wang, M. M. Wilde, and Y. Su, Quantifying the magic of quantum channels, New J. Phys. 21, 103002 (2019).

[27] G. Gour and C. M. Scandolo, The Entanglement of a Bipartite Channel, arXiv:1907.02552v3 (2019).

[28] S. Büml, S. Das, X. Wang, and M. M. Wilde, Resource Theory of Entanglement for Bipartite Quantum Channels, arXiv:1907.04181 (2020).

[29] K. Fang, O. Fawzi, R. Renner, and D. Sutter, Chain Rule for the Quantum Relative Entropy, Phys. Rev. Lett. 124, 100501 (2020).

[30] See the Supplemental Material at http://link.aps.org/supple mental/10.1103/PRXQuantum.2.010313 for details of the technical proofs of the theorems presented in the main text.

[31] G. Chiribella, G. M. D'Ariano, and P. Perinotti, Transforming quantum operations: Quantum supermaps, EPL (Europhys. Lett.) 83, 30004 (2008).

[32] J. Burniston, M. Grabowecky, C. M. Scandolo, G. Chiribella, and G. Gour, ssNecessary and sufficient conditions 
on measurements of quantum channels, Proc. R. Soc. A: Math., Phys. Eng. Sci. 476, 20190832 (2020).

[33] N. Datta, Max-relative entropy of entanglement, alias log robustness, Int. J. Quantum Inf. 7, 475 (2009).

[34] D. Petz, Quantum Information Theory and Quantum Statistics, Theoretical and Mathematical Physics (Springer, 2008).

[35] E. Chitambar and G. Gour, Quantum resource theories, Rev. Mod. Phys. 91, 025001 (2019).

[36] K. Matsumoto, Reverse Test and Characterization of Quantum Relative Entropy, arXiv:1010.1030 (2018).

[37] H. Wilming, R. Gallego, and J. Eisert, Axiomatic characterization of the quantum relative entropy and free energy, Entropy 19, 241 (2017).

[38] D. Blackwell, Equivalent comparisons of experiments, Ann. Math. Statist. 24, 265 (1953).

[39] J. Arthur F. Veinott, Least d-majorized network flows with inventory and statistical applications, Manage. Sci. 17, 547 (1971).

[40] G. Dahl, Matrix majorization, Linear Algebra Appl. 288, 53 (1999).
[41] M. Horodecki and J. Oppenheim, Fundamental limitations for quantum and nanoscale thermodynamics, Nat. Commun. 4, 2059 (2013).

[42] A. W. Marshall, I. Olkin, and B. Arnold, Inequalities: Theory of Majorization and Its Applications (Springer, 2011).

[43] K. Fang (private communication).

[44] J. Watrous, The Theory of Quantum Information (Cambridge University Press, Cambridge, 2018).

[45] S. Das and M. M. Wilde, Quantum reading capacity: General definition and bounds, IEEE Trans. Inf. Theory 65, 7566 (2019).

[46] S. Das and M. M. Wilde, Quantum rebound capacity, Phys. Rev. A 100, 030302 (2019).

[47] M. Takeoka and M. M. Wilde, Optimal Estimation and Discrimination of Excess Noise in Thermal and Amplifier Channels, arXiv:1611.09165 (2016).

[48] D. Aharonov, A. Kitaev, and N. Nisan, in Proceedings of the Thirtieth Annual ACM Symposium on Theory of Computing, STOC '98 (Association for Computing Machinery, New York, 1998), p. 20. 